\title{
The reactive-telegraph equation and a related kinetic model
}

\author{
Christopher Henderson| and Panagiotis E. Souganidiff
}

June 22, 2021

\begin{abstract}
We study the long-range, long-time behavior of the reactive-telegraph equation and a related reactive-kinetic model. The two problems are equivalent in one spatial dimension. We point out that the reactive-telegraph equation, meant to model a population density, does not preserve positivity in higher dimensions. In view of this, in dimensions larger than one, we consider a reactive-kinetic model and investigate the long-range, long-time limit of the solutions. We provide a general characterization of the speed of propagation and we compute it explicitly in one and two dimensions. We show that a phase transition between parabolic and hyperbolic behavior takes place only in one dimension. Finally, we investigate the hydrodynamic limit of the limiting problem.
\end{abstract}

\section{Introduction}

The Fisher-KPP equation

$$
u_{t}=u_{x x}+u(1-u)
$$

is the classical model used to study the spread of a population in an environment. Being based on the heat equation, the Fisher-KPP equation exhibits infinite speed of propagation. Indeed the population density is non-zero everywhere for any positive time even if the initial data is compactly supported.

One approach to remove this unphysical behavior is to look at the reactive-telegraph equation

$$
\tau \rho_{t t}+(1-\tau(1-2 \rho)) \rho_{t}=\Delta \rho+\rho(1-\rho) .
$$

This may be stated with more general non-linearities $F(\rho)$ in place of $\rho(1-\rho)$ and $F^{\prime}(\rho)$ in place of $1-2 \rho$. In one dimension, this is equivalent, for $\rho=\frac{p^{-}+p^{+}}{2}$, to the kinetic system

$$
\left\{\begin{array}{l}
p_{t}^{+}+\frac{1}{\sqrt{\tau}} p_{x}^{+}=\frac{1}{2 \tau}\left(p^{-}-p^{+}\right)+\frac{1}{2} \rho(1-\rho), \\
p_{t}^{-}-\frac{1}{\sqrt{\tau}} p_{x}^{-}=\frac{1}{2 \tau}\left(p^{+}-p^{-}\right)+\frac{1}{2} \rho(1-\rho) .
\end{array}\right.
$$

The solution $\rho$ is a population density, while $p^{ \pm}$represents the density of those individuals moving with velocity \pm 1 . The positive parameter $\tau$ is related to the relaxation time, which depends on

\footnotetext{
${ }^{*}$ Corresponding author, Department of Mathematics, The University of Chicago, 5734 S. University Avenue, Chicago, IL 60637, E-mail: henderson@math.uchicago.edu

${ }^{\dagger}$ Department of Mathematics, The University of Chicago, 5734 S. University Avenue, Chicago, IL 60637, E-mail: souganidis@math. uchicago.edu
} 
the mean run length between changes in velocity. A small sample of the more applied literature addressing these models, including the interpretation of $\tau$ can be found in the works of Fedotov [17, Hillen and Othmer [18, Holmes [19], Horsthemke [20], Kac [21], Méndez, Fort, and Farjas [24], and Ortega, Fort, and Méndez [25].

We discuss, in particular the work in [17], where the author proposed (1.1) as a model for population dynamics in more than one dimension, deriving it from a general transport model with the flux given by convolution of the gradient with a kernel, and formally analyzed the long-range, long-time behavior of the solution with arguments similar to those used to rigorously study the same problem for the Fisher-KPP equation. However, to even write down this formal analysis it is necessary to know that the solutions to (1.1) preserve the sign, that is if they start nonnegative, they remain nonnegative.

Our first result is to show that, in general, such formal computations cannot be justified when $n \geq 2$. Indeed, solutions to (1.1) need not remain positive. In other words, it is not possible to rule out negative population densities, which are unphysical.

Theorem 1.1. Assume $n=1$ and fix $\rho_{0} \in C^{1}(\mathbb{R}) \cap L^{1}(\mathbb{R})$ with $0 \leq \rho_{0} \leq 1$. If $\rho$ is the solution of (1.1) with initial data $\rho(\cdot, 0)=\rho_{0}$ and $\rho_{t}(\cdot, 0)=0$, then $0 \leq \rho(x, t) \leq 2$ for all $(x, t) \in \mathbb{R} \times[0, \infty)$.

If $n \geq 2$, there exists $\rho_{0} \in C^{\infty}\left(\mathbb{R}^{n}\right)$ with $0 \leq \rho_{0} \leq 1$ and $\left(x_{0}, t_{0}\right) \in \mathbb{R}^{n} \times \mathbb{R}_{+}$such that, if $\rho$ solves (1.1) with initial data $\rho(\cdot, 0)=\rho_{0}$ and $\rho_{t}(\cdot, 0)=0$, then $\rho\left(x_{0}, t_{0}\right)<0$.

We discuss first why (1.1) preserves positivity when $n=1$. In this case, 1.1) is equivalent to (1.2), which preserves positivity because, roughly, $\rho$ is controlled by $p$ from below. Neither the equivalence of the model (1.1) to a kinetic equation nor the fact that $\rho$ is controlled below by $p$ holds in higher dimensions.

As is evident in the proof of Theorem 1.1, 1.1) does not preserve positivity for a wide class of non-linearities $F$. The essential ingredients of the proof are that the equation is well-defined for short times for data in a "nice" enough Sobolev space and that $F$ is piecewise $C^{1}$ near zero with $F(0)=0$. In particular, the second claim in Theorem 1.1 is due to the properties of the wave operator and not the non-linearity $\rho(1-\rho)$.

In view of the discussion above, the reactive-telegraph equation allows for negative population densities, suggesting that it may not be an appropriate biological model in some settings. As such, we restrict our focus to the following generalization of the kinetic system $(1.2)$

$$
p_{t}+a_{n, \tau} v \cdot D p=\frac{1}{\tau}(\rho-p)+\rho(1-\rho)_{+} \text {in } \mathbb{R}^{n} \times S^{n-1} \times \mathbb{R}_{+},
$$

where $D$ is the spatial gradient, $p$ is the density of individuals moving with velocity $v \in S^{n-1}$,

$$
\rho(x, t):=f_{S^{n-1}} p(x, v, t) d v
$$

is the population density,

$$
a_{n, \tau}:=\sqrt{n / \tau}
$$

is the speed of pure transport, $S^{n-1}$ is the unit sphere in $\mathbb{R}^{n}, \mathbb{R}_{+}:=(0, \infty), f$ denotes the normalized integral such that $f_{S^{n-1}} d x=1$, and $x_{+}:=\max (x, 0)$.

We make a few general comments regarding (1.3). First, although the reactive-telegraph equation (1.1) is equivalent to a kinetic model like (1.3) (cf. 1.2) in one dimension, there is no rigorous 
connection between the two in higher dimensions. Second, we use the non-linearity $\rho(1-\rho)_{+}$so that the model preserves positivity ${ }^{1}$ and retains the fundamental aspects of the logistic one, $\rho(1-\rho)$. In particular, it represents the physical assumption that growth and competition depend only on the total population at a particular location and do not depend on velocity. Lastly, the $\sqrt{n}$ factor in $a_{n, \tau}$ is to fix the hydrodynamic limit $\tau \rightarrow 0$ as the classical Fisher-KPP equation regardless of the dimension. This is discussed later in the paper (see Proposition 1.6).

By analogy with the Fisher-KPP equation, we expect the population to spread linearly-in-time. In situations like this, where a front ${ }^{2}$ is expected to move at an approximately constant speed, it is standard to use the hyperbolic long-range, long-time limit, see Barles, Evans and Souganidis [2], Evans and Souganidis [16], and Majda and Souganidis [23] and the large body of literature citing these works. This limit corresponds to scaling space and time by the same large parameter in order to capture this linear-in-time propagation while ignoring fluctuations and short-time behavior.

As such, we use the hyperbolic scaling $(x, v, t) \mapsto(x / \epsilon, v, t / \epsilon)$ and consider the rescaled function

$$
p^{\epsilon}(x, v, t):=p(x / \epsilon, v, t / \epsilon),
$$

which solves

$$
p_{t}^{\epsilon}+a_{n, \tau} v \cdot D p^{\epsilon}=\frac{1}{\epsilon \tau}\left(\rho^{\epsilon}-p^{\epsilon}\right)+\frac{1}{\epsilon} \rho^{\epsilon}\left(1-\rho^{\epsilon}\right)_{+} \text {in } \mathbb{R}^{n} \times S^{n-1} \times \mathbb{R}_{+},
$$

with

$$
\rho^{\epsilon}(x, t):=f_{S^{n-1}} p^{\epsilon}(x, v, t) d v
$$

We study the behavior, as $\epsilon \rightarrow 0$, of $p^{\epsilon}$ and $\rho^{\epsilon}$ with initial datum

$$
p^{\epsilon}(\cdot, \cdot, 0)=p_{0} \text { on } \mathbb{R}^{n} \times S^{n-1} \times\{0\}
$$

such that

$$
\left\{\begin{array}{l}
0 \leq p_{0} \leq 1, \text { and there exists an open, Lipschitz domain } G_{0} \subset \mathbb{R}^{n} \text { so that } \\
G_{0}:=\left\{x \in \mathbb{R}^{n}: \inf _{v} p_{0}(x, v)>0\right\} \text { and } G_{0}^{c}=\left\{x \in \mathbb{R}^{n}: \sup _{v} p_{0}(x, v)=0\right\}
\end{array}\right.
$$

In order to investigate the propagation properties of $p^{\epsilon}$ and $\rho^{\epsilon}$ as $\epsilon \rightarrow 0$, we use the classical HopfCole transform $p^{\epsilon}=\exp \left(J^{\epsilon} / \epsilon\right)$, which is a standard tool in the study of front propagation [2, 16, 23], and study first the behavior, as $\epsilon \rightarrow 0$, of the $J^{\epsilon}$ 's, which solve

$$
\left\{\begin{aligned}
J_{t}^{\epsilon}+a_{n, \tau} v \cdot D J^{\epsilon} & =\frac{1}{\tau}\left(f_{S^{n-1}} e^{\frac{1}{\epsilon}\left(J^{\epsilon}\left(x, v^{\prime}, t\right)-J^{\epsilon}(x, v, t)\right)} d v^{\prime}-1\right) \\
& +f_{S^{n-1}} e^{\frac{1}{\epsilon}\left(J^{\epsilon}\left(x, v^{\prime}, t\right)-J^{\epsilon}(x, v, t)\right)} d v^{\prime}\left(1-\rho^{\epsilon}\right)_{+} \text {in } \mathbb{R}^{n} \times S^{n-1} \times \mathbb{R}_{+} .
\end{aligned}\right.
$$

Note that it is possible to use this transformation since $p^{\epsilon} \geq 0$ in $\mathbb{R}^{n} \times S^{n-1} \times \mathbb{R}_{+}$. This is shown to be the case in Lemma 2.1 if 1.7 holds.

In what follows, when necessary to signify the dependence on $\tau$, we write $J^{\epsilon, \tau}, J^{\tau}$ and $H^{\tau}$ instead of $J^{\epsilon}, J$ and $H$.

\footnotetext{
${ }^{1}$ See Lemma 2.1 for a proof of the preservation of positivity and see Section 6 for a discussion of a related model with the logistic non-linearity $\rho(1-\rho)$ that does not preserve positivity, which suggests that $\rho(1-\rho)_{+}$is a better choice of non-linearity.

${ }^{2}$ By "front," we mean the area between where $\rho$ approximately takes the value 0 and the value 1.
} 
Theorem 1.2. Assume (1.7) and let $J^{\epsilon}$ solve (1.8) with initial data $J^{\epsilon}(\cdot, \cdot, 0)=\epsilon \log p_{0}$ on $\mathbb{R}^{n} \times$ $S^{n-1}$. Then, for each $\tau>0$, there exists a concave, rotationally invariant function $H: \mathbb{R}^{n} \rightarrow \mathbb{R}$, defined in (3.4), such that, as $\epsilon \rightarrow 0$ and uniformly in $v$ and locally uniformly in $\mathbb{R}^{n} \times \mathbb{R}_{+}, J^{\epsilon}$ converges to $J$, the unique solution to

$$
\max \left(J_{t}+H(D J), J\right)=0 \quad \text { in } \mathbb{R}^{n} \times \mathbb{R}_{+} \quad J(\cdot, 0)= \begin{cases}-\infty & \text { in } \bar{G}_{0}^{c}, \\ 0 & \text { in } G_{0} .\end{cases}
$$

We make a few brief comments on some technical aspects of Theorem 1.2. First, the limiting function $J$ does not depend on $v$. Second, when $p_{0}$ is zero, we define $\log p_{0}$ to be the extended real value $-\infty$. Lastly, by the locally uniform convergence of $J^{\epsilon}$ to $J$, we mean that this convergence is uniform on compact sets $K$ such that either $K \subset \operatorname{Int}\{J=-\infty\}$ or $K \subset \operatorname{Int}\{J<-\infty\}$.

Knowing Theorem 1.2 we then infer the following spreading behavior of $\rho^{\epsilon}$.

Theorem 1.3. Suppose that (1.7) holds and that $J$ is the solution to (1.9). Then, as $\epsilon \rightarrow 0$ and locally uniformly in $\{J<0\}, \lim _{\epsilon \rightarrow 0} \rho^{\epsilon}(x, t)=0$.

If $\tau \leq 1$, then, as $\epsilon \rightarrow 0$ and locally uniformly in $\operatorname{Int}\{J=0\}, \lim _{\epsilon \rightarrow 0} \rho^{\epsilon}(x, t)=1$.

If $\tau>1$, then, as $\epsilon \rightarrow 0$ and locally uniformly in $\operatorname{Int}\{J=0\}, \liminf _{\epsilon \rightarrow 0} \rho^{\epsilon}(x, t) \geq 1$.

Before discussing the proof of Theorem 1.3 , we mention the reason that there is a distinction between $\tau>1$ and $\tau \leq 1$. When $\tau<1$, it is possible to bound $p$ by 1 by using a maximum principle-type argument. On the other hand, when $\tau \geq 1$, it is shown in Lemma 2.1 that upper bound is order $(1+\tau)^{2} / 4 \tau$. The proof of Theorem 1.3 yields that $\liminf _{\epsilon \rightarrow 0} \rho^{\epsilon}(x, t) \geq 1$ on $\operatorname{Int}\{J=0\}$. When $\tau<1$, the bound above gives us immediately that $\lim _{\sup _{\epsilon \rightarrow 0}} \rho^{\epsilon}(x, t) \leq 1$, yielding the exact value of the limit. When $\tau \geq 1$, this argument does not work.

To prove the asymptotic results, we use the classical half-relaxed limits of Barles and Perthame [3] along the lines of [2, 16] and a modification due to Barles and Souganidis [4, which allows us to side-step the technical difficulty that, due to the finite speed of propagation in kinetic equations, the $J^{\epsilon}$ 's take the value $-\infty$. The combination of the half-relaxed limits and the technique of [4] is that, roughly, when the limiting Hamilton-Jacobi equation represents a distance function, no a priori bounds or regularity are needed to pass to the limit $\epsilon \rightarrow 0$.

The Hamiltonian $H$ in Theorem 1.2 is the same one found by Bouin and Calvez [6] and Caillerie [12] in a related context since the linearized equations are the same.

Since $H$ in 1.9 is space-time homogeneous, it follows from [16, 23] that

$$
J(x, t)=\min (I(x, t), 0),
$$

where $I$ is the solution to

$$
I_{t}+H(D I)=0 \text { in } \mathbb{R}^{n} \times \mathbb{R}_{+}, \quad I(\cdot, 0)=\left\{\begin{array}{l}
-\infty \text { in } \bar{G}_{0}^{c}, \\
0 \text { in } G_{0} .
\end{array}\right.
$$

Let $L$ be the concave dual of $H$. Then, see, for example, Lions [22],

$$
I(x, t)=t \sup \left\{L\left(\frac{x-y}{t}\right): y \in G_{0}\right\} .
$$

As mentioned in Theorem 1.2, $H$ is concave and rotationally invarant. From this it follows that $H$ is radially decreasing; this can also be seen from the explicit formula (3.4). As a result, $L$ has the 
same properties. Taking some liberty with notation, we write $L(q)=L(|q|)$. It then follows that, for $t \in \mathbb{R}_{+}$,

$$
\left\{x \in \mathbb{R}^{n}: J(x, t)<0\right\}=\left\{x \in \mathbb{R}^{n}: \sup _{y \in G_{0}} L\left(\frac{x-y}{t}\right)<0\right\}=\left\{x \in \mathbb{R}^{n}: L\left(\frac{d\left(x, G_{0}\right)}{t}\right)<0\right\},
$$

where $d\left(x, G_{0}\right)$ is the usual distance from the point $x$ to the closed set $G_{0}$.

In view of Theorem 1.3 , it is clear that the front is $\partial\left\{x \in \mathbb{R}^{n}: J(x, t)<0\right\}$. From the discussion above, we see that

$$
\partial\left\{x \in \mathbb{R}^{n}: J(x, t)<0\right\}=\partial\left\{x \in \mathbb{R}^{n}: I(x, t)<0\right\}=\left\{x \in \mathbb{R}^{n}: L\left(\frac{d\left(x, G_{0}\right)}{t}\right)=0\right\} .
$$

The next result, which holds for $n \geq 2$, provides a characterization of the 0-level set of $L$ and, hence, the speed of propagation in terms of a global property of the Hamiltonian.

Let

$$
c_{n, \tau}:=-\sup _{q \in \mathbb{R}^{n}} \frac{H(q)}{|q|} .
$$

The claim is:

Proposition 1.4. Assume (1.7) and $n \geq 2$. Then $c_{n, \tau}$ is achieved, $c_{n, \tau}<a_{n, \tau}$, and the front is the set $\left\{x \in \mathbb{R}^{n}: d\left(x, G_{0}\right)=c_{n, \tau} t\right\}$.

Since $c_{n, \tau}<a_{n, \tau}$, it follows that, for $n \geq 2$, the front moves with velocity slower than that of pure transport. Following [8], we call this behavior parabolic to distinguish it from the hyperbolic one, which is observed, as discussed below, for $n=1$, when $\tau \geq 1$.

To heuristically justify the term "parabolic," we return to the unscaled problem (1.3) and discuss the behavior of $\rho$ when $\rho_{0}$ has compact support. Indeed, due to the kinetic nature of (1.3), the support of $\rho$ propagates with the speed of the pure transport $a_{n, \tau}$. Proposition 1.4 , however, implies that the set on which $\rho$ is approximately 1 propagates at speed $c_{n, \tau}$. This difference suggests that $\rho$ has long tails connecting 0 and 1 . As a result the profile resembles that of solutions to the Fisher-KPP equation, whose long tails are caused by the infinite speed of propagation in the heat equation. See Figure 1 for a cartoon picture of this, and see [8] for an explicit construction where the long tails are observed.

When $n=1$ and $n=2$ it is possible to explicitly compute $H$ and, hence, the speed of propagation. This is the contents of the proposition below. We point out that the speed $c_{1, \tau}$ is the one given in [17] and is the speed of the traveling waves constructed by Bouin, Calvez, and Nadin in [8].

Proposition 1.5. Assume (1.7). Then

$$
c_{\tau, 1}=\left\{\begin{array}{l}
\frac{2}{1+\tau} \quad \text { if } \tau \leq 1, \\
\frac{1}{\sqrt{\tau}} \quad \text { if } \tau \geq 1,
\end{array} \quad \text { and } \quad c_{\tau, 2}=\frac{\sqrt{2(2+\tau)}}{1+\tau} .\right.
$$

When $n=1$ and $\tau \geq 1, a_{1, \tau}=c_{1, \tau}$. We refer to this behavior as "hyperbolic". To heuristically justify this term, we again return to the unscaled problem 1.3 and consider initial data $\rho_{0}$ with compact support. Following the discussion above, we see that the support of $\rho$ and the set on which $\rho$ approximately takes the value 1 move with the same speed. In particular, we do not 

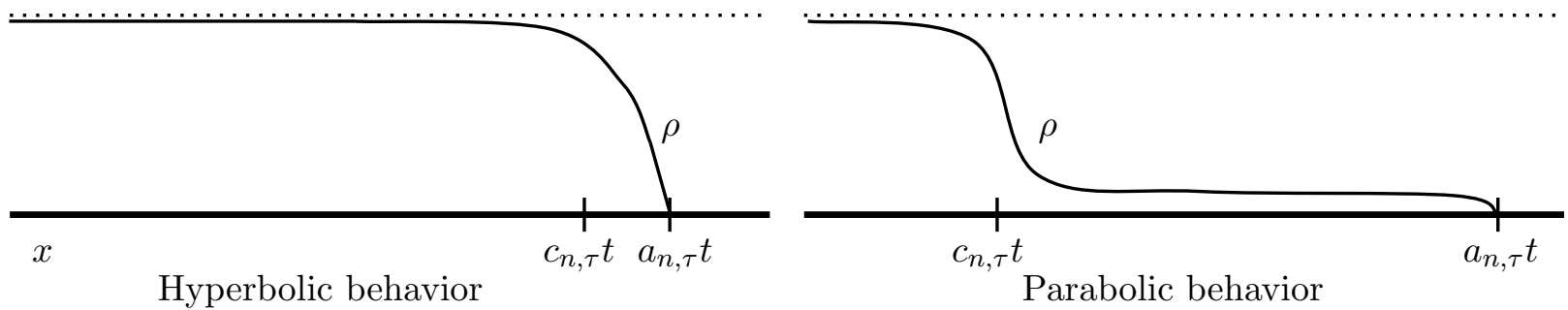

Figure 1: Cartoon pictures showing the difference between the hyperbolic behavior (the graph on the left) seen in one spatial dimension when $\tau \geq 1$ and the parabolic behavior (the graph on the right) seen otherwise. The key distinction is, in the parabolic behavior, the formation of long tails connecting the boundary of the support of $\rho$, which progress with speed $a_{n, \tau}$, with the location of the front, which progresses with speed $c_{n, \tau}$, which is strictly less than $a_{n, \tau}$.

see the formation of long tails as in the discussion above. Once more, see Figure 1 for a cartoon representation of this behavior and see [8] for an explicit construction where one sees this behavior.

It follows from the last two propositions that there is a phase transition, depending on $\tau$, between parabolic and hyperbolic behavior only in one dimension. We now heuristically explain the reason for this. In order to exhibit hyperbolic behavior, growth due to the reaction must "keep up" with pure transport. That this is possible in only $\mathbb{R}^{1}$ is related to the fact that $p$ controls $\rho$ from below; that is, for any $v \in S^{0}=\{-1,1\}, p(x, v, t) / 2 \leq \rho(x, t)$ for all $(x, t)$. In particular, the non-local reaction term $\rho(1-\rho)_{+}$can be bounded below by a local term when $\rho$ is small. This is not possible in higher dimensions.

Formally, taking the limit $\tau \rightarrow 0$ in (1.1), we recover the Fisher-KPP equation $u_{t}=\Delta u+u(1-u)$. In addition, solutions of 1.3 formally converge, as $\tau \rightarrow 0$, to the Fisher-KPP equation regardless of dimension; see, for example, the discussion of Cuesta, Hittmeir, and Schmeiser in [14] and also Section 3 of [18].

We prove that the limits may be taken in the reverse order to obtain the same limiting object. Indeed, fix $u_{0}$ such that $G_{0}=\left\{u_{0}>0\right\}$ and let $u^{\epsilon}$ be the solution to

$$
u_{t}^{\epsilon}=\epsilon \Delta u^{\epsilon}+\epsilon^{-1} u^{\epsilon}\left(1-u^{\epsilon}\right) \text { in } \mathbb{R}^{n} \times \mathbb{R}_{+} \quad u^{\epsilon}(\cdot, 0)=u_{0} \text { on } \mathbb{R}^{n} .
$$

It is well-known (see [16]) that, as $\epsilon \rightarrow 0$ and locally uniformly in $(x, t), z^{\epsilon}:=\epsilon \log u^{\epsilon}$ converges to the unique solution $z$ of

$$
\max \left\{z_{t}-|D z|^{2}-1, z\right\}=0 \text { in } \mathbb{R}^{n} \times \mathbb{R}_{+} \quad z(\cdot, 0)=\left\{\begin{array}{l}
0 \text { on } G_{0}, \\
-\infty \text { on } \bar{G}_{0}^{c} .
\end{array}\right.
$$

We show that, as $\tau \rightarrow 0, J^{\tau} \rightarrow z$, implying that $\lim _{\tau \rightarrow 0} \lim _{\epsilon \rightarrow 0} J^{\tau, \epsilon}=z$. This is what one would expect in view of the discussion above, which formally gives that $\lim _{\epsilon \rightarrow 0} \lim _{\tau \rightarrow 0} J^{\tau, \epsilon}=z$.

Proposition 1.6. Assume (1.7) and let $J^{\tau}$ and $z$ be respectively the unique solutions to (1.9) and (1.14). Then, as $\tau \rightarrow 0$ and locally uniformly in $\mathbb{R}^{n} \times \mathbb{R}_{+}, J^{\tau} \rightarrow z$.

The convergence in Proposition 1.6 essentially follows from the formal convergence of $H^{\tau}$ to $-|\cdot|^{2}-1$. As seen explicitly in the proof, without the $\sqrt{n}$ factor in $(1.4)$, there would be a factor $n$ in the equation for $\lim _{\tau \rightarrow 0} J^{\tau}$. This is related to the fact that the variance of any unit vector over $S^{n-1}$ is $1 / n$. We mention briefly that, in the equation $(1.3)$, the $\sqrt{n}$ factor can be added or removed by simply scaling in $x$ and therefore does not affect our analysis. 
Next we discuss briefly related works. As mentioned above, the one dimensional problems have been considered from the modeling perspective in [18, 19, 21]. The reactive-telegraph model (1.1) has only been rigorously studied in the one dimensional setting. We point out, in particular, the work of Bouin, Calvez, and Nadin [8], in which they prove existence and stability of traveling waves in a weighted space. In addition, there is [17] which was discussed earlier. There has been more work recently on related kinetic equations in one dimension. Bouin and Calvez [6] obtained convergence to a limiting equation for the linearization of (1.3) assuming that $J_{0}$ is Lipschitz and bounded. Later Bouin, Calvez, and Nadin [9], and Bouin, Calvez, Grenier, and Nadin [7] showed acceleration in a kinetic-reactive equation similar to our setting but where the velocity space is unbounded and the reaction term is replaced by $\rho(M(v)-p)$. In higher dimensions, the kinetic model studied here with $\rho(1-\rho)$ replaced by $\rho(M(v)-p)$ was investigated by Caillerie [12. In this work Caillerie performs a limiting procedure with well-prepared initial data and identifies the same Hamiltonian as the one we find above 3 .

Finally, during the write-up of this work, we became aware of a parallel work by Bouin and Caillerie [5] which has some similarity to the present one. In [5], the authors investigate a related kinetic system with reaction term $\rho(M(v)-p)$ and with general dispersal kernel $M(v)$. The authors consider the long-range, long-time limit of this equation, the question of propagation in the unscaled equation, and the existence of traveling waves. There are many differences between the two papers. First, in the long-range, long-time limit, Bouin and Caillerie consider well-prepared initial data for $J^{\epsilon}$, which corresponds to initial conditions that are exponentially decaying (in contrast to the compactly supported initial conditions considered here and widely considered in the front propagation literatur $5^{5}$. Second, the equation considered by Bouin and Caillerie enjoys the maximum principle. These two facts simplify many technical details in the long-range, long-time limit. Also, the questions considered in the present work about the appropriateness of the reactive-telegraph equation, the difference in behavior with the non-linearity $\rho(1-\rho)$ versus $\rho(1-\rho)_{+}$, and the existence and dependence on dimension of the phase transition in $\tau$ are not considered in [5]. On the other hand, the work of Bouin and Caillerie considers a much broader class of kinetic models, which will be useful in studying a variety of biological models.

\section{Organization of the paper}

The paper is organized into six main parts. In Section 2 we present a preliminary lemma that gives an upper bound for $J^{\epsilon}$ that is independent of $\epsilon$ and prove the convergence part of Theorem 1.3 . The Hamiltonian and its properties as well as the proof of Theorem 1.2 are given in Section 3 . In Section 4 we prove Proposition 1.4 and Proposition 1.5. In Section 5 we show that we may take the hydrodynamic limit $\tau \rightarrow 0$ to recover the Hamilton-Jacobi equation of the Fisher-KPP equation. In Section 6 we prove Theorem 1.1 and discuss an example of a related kinetic system with logistic reaction term which does not preserve positivity either. A brief Appendix is included containing computations of some non-standard integrals that are used throughout the manuscript.

\footnotetext{
${ }^{3}$ That they are related can be seen by the fact that $F(\rho)$ and $\rho(M(v)-p)$ have the same linearization when $M(v)$ is 1 for all $v \in \mathbb{S}^{n-1}$.

${ }^{4}$ The authors fix initial data for their analogue of $J^{\epsilon}$ and apparently assume that this initial data is independent of $\epsilon$ and finite everywhere. This is in contrast to the present work in which $J^{\epsilon}$ takes infinite values in $G_{0}^{c}$.

${ }^{5}$ Since the study of front propagation is the study of a new population (or other physical quantity) invading a previously unexplored area, which corresponds to $p_{0}$ being zero, this type of assumption is crucial to a full investigation. We note that this is not a mere technical detail - there are many situations where the behavior of compactly supported initial data and well-prepared initial data are quite different (see, e.g., Bramson [10, Zlatoš [27], Cabre and Roquejoffre [1]).
} 


\section{The notion of solution}

We are not making any assumptions for $(1.5)$ and $(1.8)$ to have smooth solutions. We interpret both equations as well as the limiting Hamilton-Jacobi variational inequalities and equations in the classical Crandall-Lions viscosity sense.

\section{Acknowledgements}

We wish to thank the anonymous referees for a close reading of the manuscript and their very helpful comments. CH would like to thank Jacek Jendrej for pointing out the refence [26] regarding regularity of hyperbolic equations. CH was partially supported by the National Science Foundation Research Training Group grant DMS-1246999. PS was partially supported by the National Science Foundation grants DMS-1266383 and DMS-1600129 and the Office for Naval Research Grant N00014-17-1-2095.

\section{Bounds and the proof of Theorem 1.2}

\section{The a priori bounds}

We state as a lemma the fact that (1.5) preserves positivity and yields an upper bound that is independent of $\epsilon$. This is important, since without the positivity of $p^{\epsilon}, J^{\epsilon}$ is not well-defined, while the upper bound is needed in order to study the limit $\epsilon \rightarrow 0$.

Lemma 2.1. Assume $0 \leq p_{0} \leq 1$ and let $p^{\epsilon}$ be the solution to 1.5 and 1.6 . Then, for all $t \in \mathbb{R}_{+}$,

$$
0 \leq p^{\epsilon}(\cdot, \cdot, t) \leq M_{\tau}
$$

where

$$
M_{\tau}=\left\{\begin{array}{l}
1 \text { if } \tau \leq 1 \\
\frac{(1+\tau)^{2}}{4 \tau} \quad \text { if } \tau>1
\end{array}\right.
$$

Proof. The positivity of $p^{\epsilon}$ follows from rewriting (1.5) as

$$
p_{t}^{\epsilon}+a_{n, \tau} v \cdot D p^{\epsilon}+\frac{1}{\epsilon \tau} p^{\epsilon}=\frac{1}{\epsilon \tau} \rho^{\epsilon}+\frac{1}{\epsilon} \rho^{\epsilon}\left(1-\rho^{\epsilon}\right)_{+},
$$

and observing that the right hand side of the above equation is always nonnegative.

For the upper bound, if $\tau>1$, let

$$
q^{\epsilon}:=\frac{(1+\tau)^{2}}{4 \tau}-p^{\epsilon} \text { and } \hat{\rho}^{\epsilon}:=f_{S^{n-1}} q^{\epsilon}(x, v, t) d v .
$$

It follows that

$$
q_{t}^{\epsilon}+a_{n, \tau} v \cdot D q^{\epsilon}+\frac{1}{\epsilon \tau} q^{\epsilon}=\frac{1}{\epsilon \tau} \hat{\rho}^{\epsilon}+\frac{1}{\epsilon}\left(\hat{\rho}^{\epsilon}-\frac{(1+\tau)^{2}}{4 \tau}\right)\left(\hat{\rho}^{\epsilon}-\left(\frac{(1+\tau)^{2}}{4 \tau}-1\right)\right)_{+} .
$$

Straightforward calculations yield that the right hand side above is nonnegative, which, in turn, implies that $q^{\epsilon} \geq 0$ and, hence, the claim.

When $\tau \leq 1$ the proof is similar, so we omit the details. 


\section{Propagation}

We present the proof of Theorem 1.2 , which is an immediate consequence of Theorem 1.3 .

Proof of Theorem 1.2. Fix any $\left(x_{0}, t_{0}\right) \in\{J<0\}$ with $t_{0}>0$. Since $J^{\epsilon}$ converges locally uniformly in $x, t$ and uniformly in $v$ to $J$, then, for all $\epsilon$ sufficiently small, we have

$$
\sup _{v \in S^{n-1}} J^{\epsilon}\left(x_{0}, v, t_{0}\right) \leq J\left(x_{0}, t_{0}\right) / 2<0 .
$$

It follows that

$$
\rho^{\epsilon}\left(x_{0}, t_{0}\right)=f_{S^{n-1}} p^{\epsilon}\left(x_{0}, v, t_{0}\right) d v \leq \sup _{v \in S^{n-1}} p^{\epsilon}\left(x_{0}, v, t_{0}\right)=e^{\frac{1}{\epsilon} \sup _{v \in S^{n-1}} J^{\epsilon}\left(v, x_{0}, t_{0}\right)} \leq e^{\frac{J\left(x_{0}, t_{0}\right)}{2 \epsilon}},
$$

and, hence, $\lim _{\epsilon \rightarrow 0} \rho^{\epsilon}\left(x_{0}, t_{0}\right)=0$; the local uniformity of the limit is immediate.

Now consider any point $\left(x_{0}, t_{0}\right) \in \operatorname{Int}\{J=0\}$ with $t_{0}>0$. Let $\phi(x, t)=-\left|t-t_{0}\right|^{2}-\left|x-x_{0}\right|^{2}$ and notice that $J-\phi$ has a strict local minimum at $\left(x_{0}, t_{0}\right)$. Hence, there exist $\left(x_{\epsilon}, t_{\epsilon}\right)$ converging to $\left(x_{0}, t_{0}\right)$ such that $\min _{v} J^{\epsilon}(x, t)-\psi$ has a local minimum at $\left(x_{\epsilon}, t_{\epsilon}\right)$. Let $v_{\epsilon}$ be such that $J^{\epsilon}\left(x_{\epsilon}, v_{\epsilon}, t_{\epsilon}\right)=$ $\min _{v} J^{\epsilon}\left(x_{\epsilon}, v, t_{\epsilon}\right)$.

Uaing (1.8), we find

$$
\phi_{t}\left(x_{\epsilon}, t_{\epsilon}\right)+a_{n, \tau} v_{\epsilon} \cdot D \phi\left(x_{\epsilon}, t_{\epsilon}\right) \geq \frac{1}{\tau}\left(\frac{\rho^{\epsilon}\left(x_{\epsilon}, t_{\epsilon}\right)}{p^{\epsilon}\left(x_{\epsilon}, v_{\epsilon}, t_{\epsilon}\right)}-1\right)+\frac{\rho^{\epsilon}\left(x_{\epsilon}, t_{\epsilon}\right)}{p^{\epsilon}\left(x_{e}, v_{\epsilon}, t_{\epsilon}\right)}\left(1-\rho^{\epsilon}\left(x_{\epsilon}, t_{\epsilon}\right)\right)_{+} .
$$

Observe that, since $\left(x_{\epsilon}, v_{\epsilon}, t_{\epsilon}\right)$ is the location of a global minimum in $v$ of $J^{\epsilon}\left(x_{\epsilon}, v_{\epsilon}, t_{\epsilon}\right)$,

$$
\frac{\rho^{\epsilon}\left(x_{\epsilon}, t_{\epsilon}\right)}{p^{\epsilon}\left(x_{e}, v_{\epsilon}, t_{\epsilon}\right)} \geq 1
$$

Moreover, an explicit computation implies that, as $\epsilon \rightarrow 0, \phi_{t}\left(x_{\epsilon}, t_{\epsilon}\right)+a_{n, \tau} v_{\epsilon} \cdot D \psi\left(x_{\epsilon}, t_{\epsilon}\right) \rightarrow 0$.

Then

$$
\lim _{\epsilon \rightarrow 0} \frac{\rho^{\epsilon}\left(x_{\epsilon}, t_{\epsilon}\right)}{p^{\epsilon}\left(x_{\epsilon}, v_{\epsilon}, t_{\epsilon}\right)}=1 \text { and } \lim _{\epsilon \rightarrow 0}\left(1-\rho^{\epsilon}\left(x_{\epsilon}, t_{\epsilon}\right)\right)_{+}=0 .
$$

The second limit above implies that $\liminf _{\epsilon \rightarrow 0} \rho^{\epsilon}\left(x_{\epsilon}, t_{\epsilon}\right) \geq 1$.

Next, recall that, for all $v \in S^{n-1}, J^{\epsilon}\left(x_{0}, v, t_{0}\right) \geq J^{\epsilon}\left(x_{\epsilon}, v_{\epsilon}, t_{\epsilon}\right)-\phi\left(x_{\epsilon}, t_{\epsilon}\right)$. Hence

$$
\begin{aligned}
\rho^{\epsilon}\left(x_{0}, t_{0}\right) & =f_{S^{n-1}} e^{\frac{J^{\epsilon}\left(x_{0}, v, t_{0}\right)}{\epsilon}} d v \geq f_{S^{n-1}} e^{\frac{J^{\epsilon}\left(x_{\epsilon}, v_{\epsilon}, t_{\epsilon}\right)+\left|x_{\epsilon}-x_{0}\right|^{2}+\left|t_{\epsilon}-t_{0}\right|^{2}}{\epsilon}} d v \\
& \geq p^{\epsilon}\left(x_{\epsilon}, v_{\epsilon}, t_{\epsilon}\right)=\left(\frac{p^{\epsilon}\left(x_{\epsilon}, v_{\epsilon}, t_{\epsilon}\right)}{\rho^{\epsilon}\left(x_{\epsilon}, t_{\epsilon}\right)}\right) \rho^{\epsilon}\left(x_{\epsilon}, t_{\epsilon}\right) .
\end{aligned}
$$

Letting $\epsilon \rightarrow 0$ and using 2.2 , we conclude that

$$
\liminf _{\epsilon \rightarrow 0} \rho^{\epsilon}\left(x_{0}, t_{0}\right) \geq 1 .
$$

Since, when $\tau \leq 1, p^{\epsilon} \leq 1$, we have $\rho^{\epsilon} \leq 1$, 2.3 yields

$$
\lim _{\epsilon \rightarrow 0} \rho^{\epsilon}\left(x_{0}, t_{0}\right)=1 \text {. }
$$




\section{The Hamiltonian, its properties and the proof of Theorem 1.3}

\section{The Hamiltonian $H$ and its properties}

To motivate the choice of the Hamiltonian $H$, we first present a formal argument about the limit of the $J^{\epsilon}$ assuming that

$$
J^{\epsilon}(x, v, t)=J(x, t)+\epsilon \eta(x, v, t)+\mathrm{o}(\epsilon) .
$$

Working in $\{J<0\}$, where we can ignore $\rho^{\epsilon}$ in $\left(1-\rho^{\epsilon}\right)_{+}$, we get from 1.8 that

$$
J_{t}+a_{n, \tau} v \cdot D J=\frac{1}{\tau}\left(f_{S^{n-1}} e^{\eta\left(x, v^{\prime}, t\right)-\eta(x, v, t)} d v^{\prime}-1\right)+f_{S^{n-1}} e^{\eta\left(x, v^{\prime}, t\right)-\eta(x, v, t)} d v^{\prime} .
$$

Since $J_{t}$ is independent of $v$, there must exist some, independent of $v$, constant $H(D J)$ so that, for all $v \in S^{n-1}$,

$$
-H(D J)+\frac{1}{\tau}+a_{n, \tau} v \cdot D J=\frac{\tau+1}{\tau} f_{S^{n-1}} e^{\eta\left(x, v^{\prime}, t\right)-\eta(x, v, t)} d v^{\prime} .
$$

The above expresssion leads to the cell (eigenvalue) problem to find, for each $p \in \mathbb{R}^{n}$, a unique constant $H(p)$ (eigenvalue) and some $\eta=\eta(v ; p)$ (eigenfunction) such that, for all $v \in S^{n-1}$,

$$
\frac{e^{\eta(v)}}{f_{S^{n-1}} e^{\eta\left(v^{\prime}\right)} d v^{\prime}}=\frac{\tau+1}{\tau} \frac{1}{-H(p)+\frac{1}{\tau}+a_{n, \tau} v \cdot p} .
$$

It follows that, if it exists, $H(p)$ must be defined implicitly by

$$
\frac{\tau}{1+\tau}=f_{S^{n-1}} \frac{d v}{-H(p)+\frac{1}{\tau}+a_{n, \tau} v \cdot p} .
$$

Consider the function $\Phi:[1, \infty) \rightarrow \overline{\mathbb{R}}_{+}$given by

$$
\Phi(s):=f_{S^{n-1}} \frac{d v}{s+v_{1}} ;
$$

here $v=\left(v_{1}, v_{2}, \ldots, v_{n}\right)$. It is immediate that $\Phi^{\prime}<0$ and $\lim _{s \rightarrow \infty} \Phi(s)=0$. Moreover, as it is shown in the Appendix, $\Phi(1)=\infty$ when $n \in\{1,2,3\}$, while $\Phi(1)<\infty$ for $n>3$.

Fix $p \in \mathbb{R}^{n} \backslash\{0\}$. Then, looking at $(3.2)$ and the properties of $\Phi$ we assert that

$$
H(p):= \begin{cases}-a_{n, \tau}|p|+\frac{1}{\tau} & \text { if } \Phi(1) \leq \frac{\tau}{1+\tau} a_{n, \tau}|p|, \\ \alpha & \text { if } \Phi(1) \geq \frac{\tau}{1+\tau} a_{n, \tau}|p|,\end{cases}
$$

where, in the latter case, $\alpha$ is the unique negative number such that

$$
\Phi\left(\frac{-\alpha+\frac{1}{\tau}}{a_{n, \tau}|p|}\right)=\frac{\tau}{1+\tau} a_{n, \tau}|p| .
$$

Note that $H$ is continuous, isotropic, that is depends only on $|p|$, and $H(0)=-1$. We note that this Hamiltonian is the same as found in [5, 12].

Next we show that (3.4) is indeed correct for every $p$, when $n \in\{1,2,3\}$, and for all $p$ such that $\Phi(1)>\frac{\tau}{1+\tau} a_{n, \tau}|p|$, if $n>3$. We present the argument in the latter case, since the discussion applies to the former. 
If $\Phi(1)>\frac{\tau}{1+\tau} a_{n, \tau}|p|$, then $\eta: S^{n-1} \rightarrow \mathbb{R}$ given by

$$
e^{\eta(u)}=\frac{\tau+1}{\tau} \frac{1}{-H(p)+\frac{1}{\tau}+a_{n, \tau} v \cdot p},
$$

with $H(p)$ given by the second alternative in (3.4), clearly satisfies $(3.1)$. If $\Phi(1)=\frac{\tau}{1+\tau} a_{n, \tau}|p|$, then the $\eta$ given in (3.6) also satisfies (3.1) for all $v \in S^{n-1}$ except $v=-p /|p|$.

It is clear that, when $\Phi(1)<\frac{\tau}{1+\tau} a_{n, \tau}|p|$, it is not possible to find such an $\eta$. In the proofs, we deal with this issue by considering "approximate" correctors, which for $\delta>0$ and appropriately chosen $\mu>0$, are given by

$$
\eta_{\mu, \delta}(v):=\frac{\tau+1}{\tau} \frac{1}{\left(\delta-H(p)+\frac{1}{\tau}+a_{n, \tau} v \cdot p\right)^{\mu}} .
$$

We now discuss the concavity of $H$. We present the argument for $n>3$, since the other cases follow similarly without having to deal with the first part of the definition of the Hamiltonian.

When $|p|>(1+\tau) \Phi(1) /\left(a_{n, \tau} \tau\right)$, the concavity is obvious from 3.4$)$. When $|p|<(1+\tau) \Phi(1) /\left(a_{n, \tau} \tau\right)$, we use 3.2 to obtain

$$
f_{S^{n-1}} \frac{D_{p} H(p)-a_{n, \tau} v}{\left(-H(p)+\frac{1}{\tau}+a_{n, \tau} v \cdot p\right)^{2}} d v=0 .
$$

Differentiating again we get

$$
f_{S^{n-1}} \frac{D_{p}^{2} H(p) d v}{\left(-H(p)+\frac{1}{\tau}+a_{n, \tau} v \cdot p\right)^{2}}=-2 f_{S^{n-1}} \frac{\left(D_{p} H(p)-a_{n, \tau} v\right) \otimes\left(D_{p} H(p)-a_{n, \tau} v\right)}{\left(-H(p)+\frac{1}{\tau}+a_{n, \tau} v \cdot p\right)^{3}} d v
$$

Fix any non-zero vector $\xi \in \mathbb{R}^{n}$. Then

$$
D_{p}^{2} H(p) \xi \cdot \xi f_{S^{n-1}} \frac{d v}{\left(-H(p)+\frac{1}{\tau}+a_{n, \tau} v \cdot p\right)^{2}}=-2 f_{S^{n-1}} \frac{\left|\left(D_{p} H(p)-a_{n, \tau} v\right) \cdot \xi\right|^{2}}{\left(-H(p)+\frac{1}{\tau}+a_{n, \tau} v \cdot p\right)^{3}} d v
$$

and concavity follows after noticing that, by its construction, $-H(p)+\frac{1}{\tau}-a_{n, \tau}|p| \geq 0$.

Finally to conclude we need to show that the concavity property is preserved across $|p|=\frac{1+\tau}{\tau a_{n, \tau}} \Phi(1)$ when $\Phi(1)<\infty$. Since $H$ is isotropic, this is immediate if we show that

$$
\limsup _{|p| \rightarrow \frac{1+\tau}{\tau a_{n}, \tau} \Phi(1)^{-}}\left|D_{p} H(p)\right| \leq \lim _{|p| \rightarrow \frac{1+\tau}{\tau a_{n, \tau}} \Phi(1)^{+}}\left|D_{p} H(p)\right|=a_{n, \tau} .
$$

To establish 3.9 we consider two cases depending on whether $f_{S^{n-1}}(1+v \cdot \hat{p})^{-2} d v$ is finite or not. Note that this quantity does not depend on $\hat{p}$, since we may simply change variables in the integral.

If $f_{S^{n-1}}(1+v \cdot \hat{p})^{-2} d v<\infty$ then, in view of $(3.8)$, we get

$$
\limsup _{|p| \rightarrow \frac{1+\tau}{\tau a_{n, \tau}} \Phi(1)^{-}}\left|D_{p} H(p)\right|=\left|f_{S^{n-1}} \frac{a_{n, \tau} v}{(1+v \cdot \hat{p})^{2}} d v\right|\left(f_{S^{n-1}} \frac{1}{(1+v \cdot \hat{p})^{2}} d v\right)^{-1} \leq a_{n, \tau} .
$$

If $f_{S^{n-1}}(1+v \cdot \hat{p})^{-2} d v=\infty$, then, using 3.8 , we find

$$
\lim _{|p| \rightarrow \frac{1+\tau}{\tau a_{n}, \tau} \Phi(1)^{-}}\left|D_{p} H(p)\right|=a_{n, \tau},
$$

since, otherwise, the left hand side of (3.8) is not finite, and the claim follows. 


\section{The half-relaxed limits and the limiting equation}

We prove Theorem 1.2. The main tools are the classical (in the theory of viscosity solutions) halfrelaxed limits [3] and the methodology of [2]. The problem is that, since $J^{\epsilon}$ takes infinite values, we do not have uniform bounds. To circumvent this we use an argument introduced in [4] to deal with this kind of difficulty.

We begin with the definition of the half-relaxed limits. Given a family $f_{\epsilon}$ of uniformly bounded functions depending only on $x$ and $t$, the half-relaxed upper and lower limits $\bar{f}$ and $\underline{f}$ are respectively

$\bar{f}(x, t):=\limsup f_{\epsilon}(x, t):=\limsup _{y \rightarrow x, s \rightarrow t, \epsilon \rightarrow 0} f_{\epsilon}(y, s)$ and $\underline{f}(x, t):=\liminf f_{\epsilon}(x, t):=\liminf _{y \rightarrow x, s \rightarrow t, \epsilon \rightarrow 0} f_{\epsilon}(y, s)$.

We also remark that, in view of Lemma 2.1 .

$$
J^{\epsilon} \leq \epsilon \log M_{\tau} \text { in } \mathbb{R}^{n} \times S^{n-1} \times \mathbb{R}_{+}
$$

Fix $A>0$ and let

$$
J_{A}^{\epsilon}:=\max \left\{J^{\epsilon},-A\right\} .
$$

Since $J_{A}^{\epsilon}$ is bounded, uniformly in $\epsilon$, from above and below by $\epsilon \log M_{\tau}$ and $-A$ respectively, we take the half-relaxed upper and lower limits of $\max _{v} J_{A}^{\epsilon}$ and $\min _{v} J_{A}^{\epsilon}$ respectively; that is, we consider $\bar{J}_{A}$ and $\underline{J}_{A}$ given by

$$
\bar{J}_{A}(x, t):=\lim \sup \max _{v} J_{A}^{\epsilon}(x, v, t) \text { and } \underline{J}_{A}(x, t):=\liminf \min _{v} J_{A}^{\epsilon}(x, v, t) .
$$

We point out that $\max _{v} J_{A}^{\epsilon}$ and $\min _{v} J_{A}^{\epsilon}$ are uniformly bounded in $\epsilon$ and do not depend on $v$. Hence, their half-relaxed limits are well-defined. Further, we note that we expect the limit of $J_{A}^{\epsilon}$ to be independent of $v$ as fluctuations in $v$ will "average out." This suggests that we lose no information in taking the maximum and minimum in $v$.

To state the next lemma we introduce some additional notation. First, $J_{A, t}$ refers to the time derivative of $J_{A}$, and this notation applies similarly to other terms derived from $J_{A}$. Second, given $g: \mathbb{R}^{n} \rightarrow \mathbb{R}, g^{\star}$ and $g_{\star}$ are respectively its upper and lower semicontinuous envelopes. Moreover,

$$
\mathbf{1}_{A}:=\left\{\begin{array}{l}
0 \text { on } G_{0} \\
-A \text { on }\left(\mathbb{R}^{n} \backslash \bar{G}_{0}\right) .
\end{array}\right.
$$

Lemma 3.1. Assume (1.7). Then:

(i) $\bar{J}_{A}$ is a (viscosity) sub-solution to

$$
\left\{\begin{array}{l}
\max \left\{\bar{J}_{A, t}+H\left(D \bar{J}_{A}\right), \bar{J}_{A},-\bar{J}_{A}-A\right\} \leq 0 \text { in } \mathbb{R}^{n} \times \mathbb{R}_{+} \\
\bar{J}_{A}(\cdot, 0) \leq\left(\mathbf{1}_{A}\right)^{\star} \text { on } \mathbb{R}^{n}
\end{array}\right.
$$

(ii) $\underline{J}_{A}$ is a (viscosity) super-solution to

$$
\left\{\begin{array}{l}
\max \left\{\underline{J}_{A, t}+H\left(D \underline{J}_{A}\right), \underline{J}_{A},-\underline{J}_{A}-A\right\} \geq 0 \text { in } \mathbb{R}^{n} \times \mathbb{R}_{+}, \\
\underline{J}_{A}(\cdot, 0) \geq\left(\mathbf{1}_{A}\right)_{\star} \text { on } \mathbb{R}^{n} .
\end{array}\right.
$$


The proofs of (3.12) and (3.13) are similar. We separate into cases based on how the Hamiltonian is defined. When $H$ is defined by the second case of (3.4), the proof is based on perturbing the test function by a small multiple of the corrector. This method is classical, dating back to the work of Evans [15] (see also [23] for another early use and [5, 6] for an application in a very similar context). On the other hand, when $H$ is defined by the first case of (3.4), additional care is required because, as per the discussion in the previous subsection, exact correctors do not exist and a few more additional arguments are need in that case. This is where the proofs of $(3.12)$ and (3.13) differ.

The proofs of parts (i) and (ii) are quite long and involved, and for the readers convenience we separate them into two proofs. We first show the proof of (i), which is slightly simpler.

Proof of Lemma 3.1(i).

\section{\# The proof of $(3.12$ for positive times:}

Since $\rho^{\epsilon} \geq 0$, it follows from 1.8 that

$$
J_{t}^{\epsilon}+a_{n, \tau} v \cdot D J^{\epsilon} \leq \frac{1}{\tau}\left(f_{S^{n-1}} e^{\frac{1}{\epsilon}\left(J^{\epsilon}\left(\cdot, v^{\prime}, \cdot\right)-J^{\epsilon}(\cdot, v, \cdot)\right)} d v^{\prime}-1\right)+f_{S^{n-1}} e^{\frac{1}{\epsilon}\left(J^{\epsilon}\left(\cdot, v^{\prime}, \cdot\right)-J^{\epsilon}(\cdot, v, \cdot)\right)} d v^{\prime} .
$$

Noting that $-A$ also solves the inequality above and using that the maximum of two sub-solutions is itself a sub-solution implies that

$$
J_{A, t}^{\epsilon}+a_{n, \tau} v \cdot D J_{A}^{\epsilon} \leq \frac{1}{\tau}\left(f_{S^{n-1}} e^{\frac{1}{\epsilon}\left(J_{A}^{\epsilon}\left(\cdot, v^{\prime}, \cdot\right)-J_{A}^{\epsilon}(\cdot, v, \cdot)\right)} d v^{\prime}-1\right)+f_{S^{n-1}} e^{\frac{1}{\epsilon}\left(J_{A}^{\epsilon}\left(\cdot, v^{\prime}, \cdot\right)-J_{A}^{\epsilon}(\cdot, v, \cdot)\right)} d v^{\prime} .
$$

Let $\phi$ be a smooth test function and assume that $\bar{J}_{A}-\phi$ has a strict maximum at $\left(x_{0}, t_{0}\right)$ and $\phi\left(x_{0}, t_{0}\right)=\bar{J}_{A}\left(x_{0}, t_{0}\right)$.

We first assume that $t_{0}>0$ and claim that

$$
\max \left\{\phi_{t}\left(x_{0}, t_{0}\right)+H\left(D \phi\left(x_{0}, t_{0}\right)\right), \bar{J}_{A}\left(x_{0}, t_{0}\right),-\bar{J}_{A}\left(x_{0}, t_{0}\right)-A\right\} \leq 0 .
$$

That $\max \left\{\bar{J}_{A}\left(x_{0}, t_{0}\right),-\bar{J}_{A}\left(x_{0}, t_{0}\right)-A\right\} \leq 0$ is an immediate consequence of the definition of $\bar{J}_{A}$ and $(3.10)$.

Next we show that

$$
\phi_{t}\left(x_{0}, t_{0}\right)+H\left(D \phi\left(x_{0}, t_{0}\right)\right) \leq 0 .
$$

To simplify the presentation and shorten some formulae in what follows we write

$$
p_{0}:=D \phi\left(x_{0}, t_{0}\right) \text { and } \hat{p}_{0}:=\frac{D \phi\left(x_{0}, t_{0}\right)}{\left|D \phi\left(x_{0}, t_{0}\right)\right|} .
$$

There are two cases.

\section{Case one: If}

$$
\Phi(1)>\frac{\tau}{1+\tau} a_{n, \tau}\left|p_{0}\right|,
$$

we consider the perturbed test $\phi^{\epsilon}=\phi+\epsilon \eta\left(v ; p_{0}\right)$ with $\eta$ given by (3.6).

Let $\left(x_{\epsilon}, t_{\epsilon}\right)$ be a maximum point of $\max _{v} J_{A}^{\epsilon}-\phi^{\epsilon}$ in a neighborhood of $\left(x_{0}, t_{0}\right)$. The use of such an approximating sequence in the theory of viscosity solutions is standard (see, for example, [1, 22]). In short, using the boundedness and continuity of $\eta$ along with the fact that $\left(x_{0}, t_{0}\right)$ is a strict local maximum of $\bar{J}_{A}-\phi$, its existence follows directly from the definition of limsup. 
Then there exist $v_{\epsilon} \in S^{n-1}$ such that $\left(x_{\epsilon}, v_{\epsilon}, t_{\epsilon}\right)$ is a maximum point of $J_{A}^{\epsilon}-\phi^{\epsilon}$ and along a subsequence, which we denote the same way, $\epsilon \rightarrow 0$, there exists $v_{0} \in S^{n-1}$ such that $\left(x_{\epsilon}, v_{\epsilon}, t_{\epsilon}\right) \rightarrow$ $\left(x_{0}, v_{0}, t_{0}\right)$ and $J_{A}^{\epsilon}\left(x_{\epsilon}, v_{\epsilon}, t_{\epsilon}\right) \rightarrow \bar{J}_{A}\left(x_{0}, t_{0}\right)$. We note that the existence of the limiting vector $v_{0} \in$ $S^{n-1}$ follows from the compactness of $S^{n-1}$.

It follows that, for $\epsilon$ small enough and all $v \in S^{n-1}$ and all $(x, t)$ in a small ball $B_{r}\left(x_{0}, t_{0}\right)$ of radius $r>0$,

$$
\phi^{\epsilon}(x, v, t)-\phi^{\epsilon}\left(x_{\epsilon}, v_{\epsilon}, t_{\epsilon}\right) \geq J_{A}^{\epsilon}(x, v, t)-J_{A}^{\epsilon}\left(x_{\epsilon}, v_{\epsilon}, t_{\epsilon}\right) .
$$

Then (3.14) implies

$$
\phi_{t}^{\epsilon}\left(x_{\epsilon}, v_{\epsilon}, t_{\epsilon}\right)+a_{n, \tau} v_{\epsilon} \cdot D \phi^{\epsilon}\left(x_{\epsilon}, v_{\epsilon}, t_{\epsilon}\right)+\frac{1}{\tau} \leq \frac{\tau+1}{\tau} f_{S^{n-1}} e^{\eta\left(v^{\prime}\right)-\eta\left(v_{\epsilon}\right)} d v^{\prime} .
$$

Using now the definition of $\eta$ in $(3.18)$ we find

$$
\phi_{t}^{\epsilon}\left(x_{\epsilon}, t_{\epsilon}\right)+a_{n, \tau} v_{\epsilon} \cdot D \phi^{\epsilon}\left(x_{\epsilon}, t_{\epsilon}\right) \leq-H\left(D \phi\left(x_{0}, t_{0}\right)\right)+a_{n, \tau} v_{\epsilon} \cdot D \phi\left(x_{0}, t_{0}\right),
$$

which, after letting $\epsilon \rightarrow 0$, gives (3.16).

Case two: If

$$
\Phi(1) \leq \frac{\tau}{1+\tau} a_{n, \tau}\left|D \phi\left(x_{0}, t_{0}\right)\right|,
$$

we observe that the previous argument cannot be repeated verbatim, since, as remarked on during the previous discussion, we do not have an exact corrector. We follow the same line of proof as above but with an additional twist to deal with this difficulty.

For $\delta>0$, we consider the "approximate" corrector $\eta_{\delta}$ given by

$$
e^{\eta_{\delta}(v)}=\frac{\tau+1}{\tau a_{n, \tau}\left|p_{0}\right|} \frac{1}{1+\delta+v \cdot \hat{p}_{0}},
$$

and note that

$$
\begin{aligned}
f_{S^{n-1}} e^{\eta_{\delta}(v)} d v & =\frac{\tau+1}{\tau a_{n, \tau}\left|p_{0}\right|} f_{S^{n-1}} \frac{1}{1+\delta+v \cdot \hat{p}_{0}} d v \\
& \leq \frac{\tau+1}{\tau a_{n, \tau}\left|p_{0}\right|} f_{S^{n-1}} \frac{1}{1+v \cdot \hat{p}_{0}}=\frac{\tau+1}{\tau a_{n, \tau}\left|p_{0}\right|} \Phi(1) \leq 1 .
\end{aligned}
$$

Consider the perturbed test function $\phi^{\delta, \epsilon}(x, v, t)=\phi(x, t)+\epsilon \eta_{\delta}(v)$. Let $\left(x_{\delta, \epsilon}, t_{\delta, \epsilon}\right)$ be a maximum point of $\max _{v} J_{A}^{\epsilon}-\phi^{\delta, \epsilon}$ in a neighborhood of $\left(x_{0}, t_{0}\right)$. Then there exists $v_{\delta, \epsilon} \in S^{n-1}$ such that $\left(x_{\delta, \epsilon}, v_{\delta, \epsilon}, t_{\delta, \epsilon}\right)$ is a maximum point of $J_{A}^{\epsilon}-\phi^{\delta, \epsilon}$ and along a subsequence, which we denote the same way, $\epsilon \rightarrow 0,\left(x_{\delta, \epsilon}, v_{\delta, \epsilon}, t_{\delta, \epsilon}\right) \rightarrow\left(x_{0}, v_{\delta, 0}, t_{0}\right)$ for some $v_{\delta, 0} \in S^{n-1}$ and $J_{A}^{\epsilon}\left(x_{\delta, \epsilon}, v_{\delta, \epsilon}, t_{\delta, \epsilon}\right) \rightarrow J_{A}^{\tau}\left(x_{0}, t_{0}\right)$. Note that the limit of the $\left(x_{\delta, \epsilon}, t_{\delta, \epsilon}\right)$ is independent of $\delta$ since $\left(x_{0}, t_{0}\right)$ is a strict local maximum of $\bar{J}_{A}-\phi$.

As before we have

$$
\phi_{t}^{\delta, \epsilon}\left(x_{\delta, \epsilon}, t_{\delta, \epsilon}\right)+a_{n, \tau} v_{\delta, \epsilon} \cdot D \phi^{\delta, \epsilon}\left(x_{\delta, \epsilon}, t_{\delta, \epsilon}\right)+\frac{1}{\tau} \leq \frac{\tau+1}{\tau} f_{S^{n-1}} e^{\eta_{\delta}\left(v^{\prime}\right)-\eta_{\delta}\left(v_{\delta, \epsilon}\right)} d v^{\prime}
$$

Using the definition of $\eta_{\delta}$ and $(3.19)$, we find

$$
\phi_{t}\left(x_{\delta, \epsilon}, t_{\delta, \epsilon}\right)+a_{n, \tau} v_{\delta, \epsilon} \cdot D \phi\left(x_{\delta, \epsilon}, t_{\delta, \epsilon}\right)+\frac{1}{\tau} \leq a_{n, \tau}\left|p_{0}\right|\left(1+\delta+v_{\delta, \epsilon} \cdot \hat{p}_{0}\right)=a_{n, \tau}\left(\left|p_{0}\right|(1+\delta)+v_{\delta, \epsilon} \cdot p_{0}\right) .
$$


After letting $\epsilon \rightarrow 0$, we obtain

$$
\phi_{t}\left(x_{0}, t_{0}\right)+a_{n, \tau} v_{\delta, 0} \cdot D \phi\left(x_{0}, t_{0}\right)+\frac{1}{\tau} \leq a_{n, \tau}\left(\left|p_{0}\right|(1+\delta)+v_{\delta, 0} \cdot p_{0}\right),
$$

and, hence,

$$
\phi_{t}\left(x_{0}, t_{0}\right)-(1+\delta) a_{n, \tau}\left|D \phi\left(x_{0}, t_{0}\right)\right|+\frac{1}{\tau} \leq 0,
$$

from which the claim follows after taking $\delta \rightarrow 0$.

\# The proof of $(3.12)$ at $t=0$ : To conclude the proof of the sub-solution property, we consider the case $t_{0}=0$. Here the first conclusion is that $\bar{J}_{A}$ satisfies the initial condition in the following weak sense:

$$
\min \left\{\max \left\{\bar{J}_{A, t}+H\left(D \bar{J}_{A}\right), \bar{J}_{A},-\bar{J}_{A}-A\right\}, \bar{J}_{A}-\left(\mathbf{1}_{A}\right)^{\star}\right\} \leq 0 \text { on } \mathbb{R}^{n} \times\{0\} .
$$

Then an argument as in [2] implies that actually

$$
\bar{J}_{A} \leq\left(\mathbf{1}_{A}\right)^{\star} \text { on } \mathbb{R}^{n} \times\{0\},
$$

and hence the claim.

To prove (3.20) we assume that, for some smooth $\phi, \bar{J}_{A}-\phi$ has a strict maximum at $\left(x_{0}, 0\right)$. Then we argue as before, that is we consider the perturbed test function we used for the proof when $t_{0}>0$. If the maximum points at the level $\epsilon$ are in $\mathbb{R}^{n} \times \mathbb{R}_{+}$for infinitely many $\epsilon$ 's, we argue exactly as before and obtain

$$
\max \left\{\phi_{t}\left(x_{0}, t_{0}\right)+H\left(D \phi\left(x_{0}, t_{0}\right)\right),-\bar{J}_{A}\left(x_{0}, t_{0}\right),-\bar{J}_{A}\left(x_{0}, t_{0}\right)-A\right\} \leq 0,
$$

otherwise we have

$$
\bar{J}_{A}\left(x_{0}, t_{0}\right) \leq\left(\mathbf{1}_{A}\right)^{\star}
$$

and, hence, the claim.

We now proceed with the proof of part (ii).

Proof of Lemma 3.1(ii).

\# The proof of $(3.13)$ for positive times: Let $\phi$ be a smooth test function and assume that $\underline{J}_{A}-\phi$ has a strict minimum at $\left(x_{0}, t_{0}\right)$ and $\phi\left(x_{0}, t_{0}\right)=\underline{J}_{A}\left(x_{0}, t_{0}\right)$.

We claim that

$$
\max \left\{\phi_{t}\left(x_{0}, t_{0}\right)+H\left(D \phi\left(x_{0}, t_{0}\right)\right), \underline{J}_{A}\left(x_{0}, t_{0}\right),-\underline{J}_{A}\left(x_{0}, t_{0}\right)-A\right\} \geq 0 .
$$

If $\underline{J}_{A}\left(x_{0}, t_{0}\right)=0$ or $\underline{J}_{A}\left(x_{0}, t_{0}\right)=-A$ the claim is true. Hence we assume that $-A<\underline{J}_{A}\left(x_{0}, t_{0}\right)<0$ and show that

$$
\phi_{t}\left(x_{0}, t_{0}\right)+H\left(D \phi\left(x_{0}, t_{0}\right)\right) \geq 0 .
$$

Again, to simplify the presentation and shorten some formulae, we use as before the notation $p_{0}$ and $\hat{p}_{0}$ for $D \phi\left(x_{0}, t_{0}\right)$ and $D \phi\left(x_{0}, t_{0}\right) /\left|D \phi\left(x_{0}, t_{0}\right)\right|$ respectively. There are two cases to consider.

Case one: If

$$
\Phi(1)>\frac{\tau}{1+\tau} a_{n, \tau}\left|p_{0}\right|,
$$

we use the perturbed test $\phi^{\epsilon}=\phi+\epsilon \eta\left(v ; p_{0}\right)$ with $\eta$ given by 3.6$)$. 
Let $\left(x_{\epsilon}, t_{\epsilon}\right)$ be a minimum point of $\min _{v} J_{A}^{\epsilon}-\phi^{\epsilon}$ in a neighborhood of $\left(x_{0}, t_{0}\right)$. Then there exist $v_{\epsilon} \in S^{n-1}$ such that $\left(x_{\epsilon}, v_{\epsilon}, t_{\epsilon}\right)$ is a minimum point of $J_{A}^{\epsilon}-\phi^{\epsilon}$ and, along a subsequence, which we denote the same way, $\epsilon \rightarrow 0$, there exists $v_{0} \in S^{n-1}$ such that $\left(x_{\epsilon}, v_{\epsilon}, t_{\epsilon}\right) \rightarrow\left(x_{0}, v_{0}, t_{0}\right)$ and $J_{A}^{\epsilon}\left(x_{\epsilon}, v_{\epsilon}, t_{\epsilon}\right) \rightarrow \bar{J}_{A}\left(x_{0}, t_{0}\right)$. We note that, for $\epsilon$ sufficiently small, $-A<J_{A}^{\epsilon}\left(x_{\epsilon}, v_{\epsilon}, t_{\epsilon}\right)<0$ and $J_{A}^{\epsilon}\left(x_{\epsilon}, v_{\epsilon}, t_{\epsilon}\right)=J^{\epsilon}\left(x_{\epsilon}, v_{\epsilon}, t_{\epsilon}\right)$, and, hence, the $J^{\epsilon}\left(x_{\epsilon}, v_{\epsilon}, t_{\epsilon}\right)$ 's are bounded away from 0 .

Then (1.8) implies

$$
\begin{aligned}
\phi_{t}^{\epsilon}\left(x_{\epsilon}, v_{\epsilon}, t_{\epsilon}\right)+ & a_{n, \tau} v_{\epsilon} \cdot D \phi^{\epsilon}\left(x_{\epsilon}, v_{\epsilon}, t_{\epsilon}\right)+\frac{1}{\tau} \\
& \geq\left(\frac{1}{\tau}+\left(1-\rho^{\epsilon}\left(x_{\epsilon}, t_{\epsilon}\right)\right)_{+}\right) f_{S^{n-1}} e^{\frac{J^{\epsilon}\left(x_{\epsilon}, v^{\prime}, t_{\epsilon}\right)-J\left(x_{\epsilon}, v_{\epsilon}, t_{\epsilon}\right)}{\epsilon}} d v^{\prime} .
\end{aligned}
$$

Since the left hand side of $(3.23)$ is bounded independently of $\epsilon$, so must be the integral term

$$
f_{S^{n-1}} e^{\left(J^{\epsilon}\left(x_{\epsilon}, v^{\prime}, t_{\epsilon}\right)-J\left(x_{\epsilon}, v_{\epsilon}, t_{\epsilon}\right)\right) / \epsilon} d v^{\prime}
$$

Finally, note that

$$
\rho^{\epsilon}\left(x_{\epsilon}, t_{\epsilon}\right)=e^{\frac{J^{\epsilon}\left(x_{\epsilon}, v_{\epsilon}, t_{\epsilon}\right)}{\epsilon}} f_{S^{n-1}} e^{\frac{J^{\epsilon}\left(x_{\epsilon}, v^{\prime}, t_{\epsilon}\right)-J^{\epsilon}\left(x_{\epsilon}, v_{\epsilon}, t_{\epsilon}\right)}{\epsilon}} d v^{\prime}
$$

Combining the last two observations and the formula above we conclude that

$$
\lim _{\epsilon \rightarrow 0} \rho^{\epsilon}\left(x_{\epsilon}, t_{\epsilon}\right)=0
$$

Returning to (3.23), we obtain

$$
\begin{aligned}
\phi_{t}^{\epsilon}\left(x_{\epsilon}, v_{\epsilon}, t_{\epsilon}\right)+ & a_{n, \tau} v_{\epsilon} \cdot D \phi^{\epsilon}\left(x_{\epsilon}, v_{\epsilon}, t_{\epsilon}\right)+\frac{1}{\tau} \\
& \geq \frac{1+\tau}{\tau} f_{S^{n-1}} e^{\frac{J^{\epsilon}\left(x_{\epsilon}, v^{\prime}, t_{\epsilon}\right)-J^{\epsilon}\left(x_{\epsilon}, v_{\epsilon}, t_{\epsilon}\right)}{\epsilon}} d v^{\prime}+\mathrm{o}(1) .
\end{aligned}
$$

Using now that $\left(x_{\epsilon}, v_{\epsilon}, t_{\epsilon}\right)$ is a minimum point of $J_{A}^{\epsilon}-\phi^{\epsilon}$, we get

$$
\phi_{t}^{\epsilon}\left(x_{\epsilon}, v_{\epsilon}, t_{\epsilon}\right)+a_{n, \tau} v_{\epsilon} \cdot D \phi^{\epsilon}\left(x_{\epsilon}, v_{\epsilon}, t_{\epsilon}\right)+\frac{1}{\tau} \geq \frac{1+\tau}{\tau} f_{S^{n-1}} e^{\eta\left(v^{\prime}\right)-\eta\left(v_{\epsilon}\right)} d v^{\prime}+\mathrm{o}(1) .
$$

The definition of $\eta$ in 3.6 then yields

$$
\phi_{t}^{\epsilon}\left(x_{\epsilon}, t_{\epsilon}\right)+a_{n, \tau} v_{\epsilon} \cdot D \phi^{\epsilon}\left(x_{\epsilon}, t_{\epsilon}\right) \geq-H\left(D \phi\left(x_{0}, t_{0}\right)\right)+a_{n, \tau} v_{\epsilon} \cdot D \phi\left(x_{0}, t_{0}\right)+\mathrm{o}(1),
$$

which, after letting $\epsilon \rightarrow 0$, gives the desired inequality.

Case two: If

$$
\Phi(1) \leq \frac{\tau}{1+\tau} a_{n, \tau}\left|p_{0}\right|,
$$

the argument needs to be modified. We recall that this may only occur when $n \geq 4$.

We consider, for $\mu \in\left(0, \mu_{c}\right)$, with $\mu_{c}:=(n-1) / 2$, and for $\delta>0$, the "approximate" corrector

$$
\eta_{\mu, \delta}(v)=\mu \log \left(\frac{1}{1+\delta+v \cdot \hat{p}_{0}}\right)
$$


The perturbed test function is now $\phi^{\mu, \delta, \epsilon}(x, v, t):=\phi(x, t)+\epsilon \eta_{\mu, \delta}(v)$. As in the previous case, let $\left(x_{\mu, \delta, \epsilon}, t_{\mu, \delta, \epsilon}\right)$ be local minima of $\min _{v} J_{A}^{\epsilon}-\phi^{\mu, \delta, \epsilon}$. Then there exists $v_{\mu, \delta, \epsilon} \in S^{n-1}$ such that $\left(x_{\mu, \delta, \epsilon}, v_{\mu, \delta, \epsilon}, t_{\mu, \delta, \epsilon}\right)$ is a minimum point of $J_{A}^{\epsilon}-\phi^{\mu, \delta, \epsilon}$. Further, along a subsequence, which we denote the same way, $\epsilon \rightarrow 0$, there exists $v_{\mu, \delta, 0} \in S^{n-1}$ such that $\left(x_{\mu, \delta, \epsilon}, v_{\mu, \delta, \epsilon}, t_{\mu, \delta, \epsilon}\right)$ converges to $\left(x_{0}, v_{\mu, \delta, 0}, t_{0}\right)$ and $J_{A}^{\epsilon}\left(x_{\mu, \delta, \epsilon}, v_{\mu, \delta, \epsilon}, t_{\mu, \delta, \epsilon}\right) \rightarrow \underline{J}_{A}\left(x_{0}, t_{0}\right)$. That $\left(x_{\delta, \epsilon}, t_{\delta, \epsilon}\right) \rightarrow\left(x_{0}, t_{0}\right)$ as $\epsilon \rightarrow 0$ is a consequence of the fact that $\left(x_{0}, t_{0}\right)$ is a strict minimum of $\underline{J}_{A}-\phi$.

The fact that (3.24) holds is proved as before. Moreover, as in (3.25), we find

$$
\begin{aligned}
\phi_{t}^{\mu, \delta, \epsilon}\left(x_{\mu, \delta, \epsilon}, v_{\mu, \delta, \epsilon}, t_{\mu, \delta, \epsilon}\right) & +a_{n, \tau} v_{\mu, \delta, \epsilon} \cdot D \phi^{\mu, \delta, \epsilon}\left(x_{\mu, \delta, \epsilon}, v_{\mu, \delta, \epsilon}, t_{\mu, \delta, \epsilon}\right)+\frac{1}{\tau}+\mathrm{o}(1) \\
& \geq \frac{1+\tau}{\tau} f_{S^{n-1}} e^{\eta_{\mu, \delta}\left(v^{\prime}\right)-\eta_{\mu, \delta}\left(v_{\mu, \delta, \epsilon}\right)} d v^{\prime}
\end{aligned}
$$

Letting $\epsilon \rightarrow 0$ in 3.27 yields

$$
\phi_{t}\left(x_{0}, t_{0}\right)+a_{n, \tau} v_{\mu, \delta, 0} \cdot D \phi\left(x_{0}, t_{0}\right)+\frac{1}{\tau} \geq \frac{1+\tau}{\tau} f_{S^{n-1}} e^{\eta_{\mu, \delta}\left(v^{\prime}\right)-\eta_{\mu, \delta}\left(v_{\mu, \delta, 0}\right)} d v^{\prime} .
$$

Using now the definition of $\eta_{\mu, \delta}$, we rewrite (3.28) as

$$
\phi_{t}\left(x_{0}, t_{0}\right)+a_{n, \tau} v_{\mu, \delta, 0} \cdot D \phi\left(x_{0}, t_{0}\right)+\frac{1}{\tau} \geq \frac{1+\tau}{\tau}\left(1+\delta+v_{\mu, \delta, 0} \cdot \hat{p}_{0}\right)^{\mu} f_{S^{n-1}} \frac{d v^{\prime}}{\left(1+\delta+v^{\prime} \cdot \hat{p}_{0}\right)^{\mu}} .
$$

Along a subsequence, which we denote in the same way, $\delta \rightarrow 0$, it follows that $v_{\mu, \delta, 0} \rightarrow v_{\mu, 0,0}$ for some $v_{\mu, 0,0} \in S^{n-1}$, and

$$
\phi_{t}\left(x_{0}, t_{0}\right)+a_{n, \tau} v_{\mu, 0,0} D \phi\left(x_{0}, t_{0}\right)+\frac{1}{\tau} \geq \frac{1+\tau}{\tau}\left(1+v_{\mu, 0,0} \cdot \hat{p}_{0}\right)^{\mu} f_{S^{n-1}} \frac{d v^{\prime}}{\left(1+v^{\prime} \cdot \hat{p}_{0}\right)^{\mu}} d v^{\prime} .
$$

It shown in the Appendix that

$$
\lim _{\mu \rightarrow \mu_{c}} f_{S^{n-1}} \frac{d v^{\prime}}{\left(1+v^{\prime} \cdot \hat{p}_{0}\right)^{\mu}} d v^{\prime}=\infty
$$

while the left hand side of 3.30 is bounded independently of $\mu$. It follows that $v_{\mu, 0,0} \rightarrow-\hat{p}_{0}$ as $\mu \rightarrow \mu_{c}$.

Hence,

$$
\phi_{t}\left(x_{0}, t_{0}\right)-a_{n, \tau}\left|D \phi\left(x_{0}, t_{0}\right)\right|+\frac{1}{\tau} \geq \liminf _{\mu \rightarrow \mu_{c}} \frac{1+\tau}{\tau}\left(1+v_{\mu, 0,0} \cdot \hat{p}_{0}\right)^{\mu} f_{S^{n-1}} \frac{d v^{\prime}}{\left(1+v^{\prime} \cdot \hat{p}_{0}\right)^{\mu}} \geq 0,
$$

and the proof is now complete in the case that $t_{0}=0$.

\# The proof of $(3.13)$ at $t=0$ : The case $t_{0}=0$ is treated very similarly to the work above; see the proof of the sub-solution property. As such, we omit it. The proof is now complete.

From the above, we now easily show that $J^{\epsilon}$ converges uniformly to the solution of $(1.9)$. In other words, we establish Theorem 1.2 .

Proof of Theorem 1.2. By construction, $\underline{J}_{A} \leq \bar{J}_{A}$. Also, since $\underline{J}_{A}$ is a super-solution to an equation that $\bar{J}_{A}$ is a sub-solution to, it follows that $\underline{J}_{A} \geq \bar{J}_{A}$, by the comparison principle (see Crandall, Lions and Souganidis [13]). Hence, we have that $\underline{J}_{A}=\bar{J}_{A}$. This, in turn, implies that $J_{A}^{\epsilon}$ converges locally uniformly in $(x, t)$ and uniformly in $v$ to $J_{A}^{\tau}=\underline{J}_{A}=\bar{J}_{A}$, which does not depend on $v$ and which solves (3.13).

On the other hand, it is easily seen that $\underline{J}_{A}=\inf \{-A, \underline{J}\}$ and $\bar{J}_{A}=\inf \{-A, \bar{J}\}$. Letting $A \rightarrow \infty$, we see that $J^{\epsilon}$ converges locally uniformly to a limit $J$ which solves $(1.9)$. 


\section{The speed of the moving front}

\section{Propagation speed when $n>1$}

Proof of Proposition 1.4. When $n>3$, the fact that $H(p) /|p|$ attains its maximum follows from the observation that, for large $|p|$,

$$
\frac{H(p)}{|p|}=\frac{1}{\tau|p|}-a_{n, \tau}>-a_{n, \tau}=\lim _{|p| \rightarrow \infty} \frac{H(p)}{|p|} .
$$

This last observation also yields that $c_{n, \tau}<a_{n, \tau}$.

SWhen $n=2,3, H$ is defined by the second alternative in (3.4) and the argument above can not be used. Instead we compute the Hamiltonians in order to conclude that $H(p) /|p|$ attains a maximum.

We first consider the case $n=2$. Using (1.4), (3.4) and the fact that, as shown in the Appendix, for $s \geq 1$,

$$
\Phi(s)=\frac{1}{\sqrt{s^{2}-1}},
$$

we find

$$
H(p)=\frac{1}{\tau}-\sqrt{\left(\frac{1+\tau}{\tau}\right)^{2}+\frac{2}{\tau}|p|^{2}} .
$$

Next we consider the case $n=3$. Using again (1.4), (3.4) and the fact that, as shown in the Appendix, for $s>1$,

$$
\Phi(s)=\frac{1}{2} \log \left(\frac{s+1}{s-1}\right)
$$

we obtain

$$
H(p)=\frac{1}{\tau}-\frac{\sqrt{3}|p|}{\sqrt{\tau}} \frac{e^{\frac{2 \sqrt{3 \tau}}{1+\tau}|p|}+1}{e^{\frac{2 \sqrt{3 \tau}}{1+\tau}|p|}-1} .
$$

In either case the claim follows after some straightforward calculations.

In view of the discussion in the introduction, to conclude the proof it suffices to show that, for each $e \in S^{n-1}, L\left(c_{n, \tau} e\right)=0$.

Let $p_{c} \in \mathbb{R}^{n} \backslash\{0\}$ be a maximizing vector in the definition of $c_{n, \tau}$, that is,

$$
c_{n, \tau}=-\frac{H\left(p_{c}\right)}{\left|p_{c}\right|}
$$

note that, in view of the isotropy of $H$, any rotation of $p_{c}$ is also a maximizing vector. Thus we may assume that $e \cdot p_{c}=-\left|p_{c}\right|$.

Since, for $q \in \mathbb{R}^{n}$,

$$
L(q)=\inf _{p \in \mathbb{R}^{n}}(q \cdot p-H(p))
$$

it is immediate that

$$
L\left(c_{n, \tau} e\right) \leq\left(-c_{n, \tau}\left|p_{c}\right|-H\left(p_{c}\right)\right)=0 .
$$

Rewriting the definition of $L$, we obtain

$$
L\left(c_{n, \tau} e\right)=c_{n, \tau} \inf _{p \in \mathbb{R}^{n}}|p|\left(e \cdot \hat{p}-\frac{1}{c_{n, \tau}} \frac{H(p)}{|p|}\right) .
$$


If $L\left(c_{n, \tau} e\right)<0$, then there exists $p \in \mathbb{R}^{n} \backslash\{0\}$ such that

$$
L\left(c_{n, \tau} e\right) \leq c_{n, \tau}|p|\left(-1-\frac{1}{c_{n, \tau}} \frac{H(p)}{|p|}\right)<0 .
$$

This implies that

$$
-c_{n, \tau}<\frac{H(p)}{|p|}
$$

which contradicts the definition of $c_{n, \tau}$. We conclude that $L\left(c_{n, \tau} e\right)=0$.

Explicit formulas for the speed for $n=1$ and $n=2$.

Proof of Proposition 1.5. We begin with the speed when $n=2$ since the proof is simple. Using Proposition 1.4 along with 4.2, find

$$
c_{2, \tau}=-\max _{p \in \mathbb{R}^{2}} \frac{H(p)}{|p|}=-\max _{p \in \mathbb{R}^{2}}\left(\frac{1}{\tau|p|}-\sqrt{\left(\frac{1+\tau}{\tau|p|}\right)^{2}+\frac{2}{\tau}}\right) .
$$

Elementary calculus yields that the maximum is attained when $|p|=(1+\tau) \sqrt{2+\tau} / \sqrt{2}$, giving

$$
c_{2, \tau}=\frac{\sqrt{2(2+\tau)}}{1+\tau}
$$

We now consider the case $n=1$. Let $\alpha=-H(p)+\tau^{-1}$. It follows from (3.4) and the fact that $S^{0}=\{-1,1\}$ that, for any $p \in \mathbb{R} \backslash\{0\}$,

$$
\frac{\frac{\alpha}{a_{n, \tau}|p|}}{\frac{\alpha^{2}}{a_{1, \tau}^{2}|p|^{2}}-1}=\frac{\tau}{1+\tau} a_{1, \tau}|p|
$$

and after rearranging this

$$
\alpha=\frac{1+\tau}{2 \tau}+\sqrt{\left(\frac{1+\tau}{2 \tau}\right)^{2}+\frac{1}{\tau}|p|^{2}}
$$

that is

$$
H(p)=\frac{1-\tau}{2 \tau}-\sqrt{\left(\frac{1+\tau}{2 \tau}\right)^{2}+\frac{1}{\tau}|p|^{2}} .
$$

Using elementary calculus, it is easy to see that $c_{1, \tau}$ is given by the formula in Proposition 1.5 . The definition of the concave dual and elementary calculus now yields

$$
L(q)= \begin{cases}-\frac{1-\tau}{2 \tau}+\frac{1+\tau}{2 \tau} \sqrt{1-\tau|q|^{2}}, & \text { if } \tau|q|^{2} \leq 1, \\ -\infty, & \text { otherwise }\end{cases}
$$

If $\tau<1, L(q)=0$ if and only if $|q|=2 /(1+\tau)$. In view of 1.13 , this implies that $c_{1, \tau}=2 /(1+\tau)$.

If $\tau \geq 1$, there is no $q \in \mathbb{R}$ such that $L(q)=0$. In this case, we notice that $L(q)>0$ when $\tau|q|^{2} \leq 1$ and $L(q)<0$ otherwise. Hence $\partial\{q \in \mathbb{R}: L(q)<0\}=\{q \in \mathbb{R}:|q|=1 / \sqrt{\tau}\}$. In view of (1.12), this implies that $c_{1, \tau}=1 / \sqrt{\tau}$, finishing the proof. 


\section{$5 \quad$ The limit $\tau \rightarrow 0$}

Let $J^{\tau}$ be the solution to $(1.9)$, recall that $J^{\tau} \leq 0$, and define

$$
J_{A}^{\tau}:=\max \left\{J^{\tau},-A\right\} .
$$

Consider the half-relaxed limits

$$
\bar{z}_{A}:=\limsup _{\tau \rightarrow 0} J_{A}^{\tau} \quad \text { and } \quad \underline{z}_{A}:=\liminf _{\tau \rightarrow 0} J_{A}^{\tau},
$$

and note that, by construction,

$$
-A \leq \underline{z}_{A} \leq \bar{z}_{A} \leq 0
$$

We prove the following lemma.

Lemma 5.1. Let $J^{\tau}$ solve (1.9) and define $\bar{z}_{A}$ and $\underline{z}_{A}$ by (5.1). Then

(i) $\underline{z}_{A}$ is a super-solution to

$$
\max \left\{\underline{z}_{A, t}-\left|D \underline{z}_{A}\right|^{2}-1, \underline{z}_{A},-A-\underline{z}_{A}\right\} \geq 0 \text { in } \mathbb{R}^{n} \times \mathbb{R}_{+} \quad \underline{z}_{A}(\cdot, 0) \geq\left(\mathbf{1}_{A}\right)_{\star} \text { on } \mathbb{R}^{n} .
$$

(ii) $\bar{z}_{A}$ is a sub-solution to

$$
\max \left\{\bar{z}_{A, t}-\left|D \bar{z}_{A}\right|^{2}-1, \bar{z}_{A},-A-\bar{z}_{A}\right\} \leq 0 \quad \text { in } \mathbb{R}^{n} \times \mathbb{R}_{+}, \quad \bar{z}_{A}(\cdot, 0) \leq\left(\mathbf{1}_{A}\right)^{\star} \quad \text { on } \mathbb{R}^{n} .
$$

We momentarily postpone the proof of Lemma 5.1 to note that the comparison principle yields $\bar{z}_{A}=\underline{z}_{A}$. Letting then $A \rightarrow \infty$ we obtain Proposition 1.6 from Lemma 5.1. The argument follows exactly as in Lemma 3.1, so we omit it.

Proof of Lemma 5.1. We prove only the claim for $\underline{z}_{A}$. The argument is similar for $\bar{z}_{A}$ with similar modifications as in the proof of Lemma 3.1 .

Fix any smooth test function $\phi$ and any point $\left(x_{0}, t_{0}\right)$ such that $\underline{z}_{A}-\psi$ has a strict local minimum of zero at $\left(x_{0}, t_{0}\right)$.

Assume that $t_{0}>0$. If $\underline{z}_{A}\left(x_{0}, t_{0}\right)=0$ or $-A$ the claim is immediate from (5.3). Hence, we need only consider the case where $\underline{z}_{A}\left(x_{0}, t_{0}\right) \in(-A, 0)$ and aim to show that

$$
0 \leq \phi_{t}\left(x_{0}, t_{0}\right)-\left|D \phi\left(x_{0}, t_{0}\right)\right|^{2}-1 .
$$

It follows from the definition of $\underline{z}_{A}$ that, as $\tau \rightarrow 0$, there exists a sequence $\left(x_{\tau}, t_{\tau}\right) \rightarrow\left(x_{0}, t_{0}\right)$ such that $J_{A}^{\tau}-\phi$ has a local minimum at $\left(x_{\tau}, t_{\tau}\right)$ and $J_{A}^{\tau}\left(x_{\tau}, t_{\tau}\right)-\phi\left(x_{\tau}, t_{\tau}\right) \rightarrow_{z_{A}}\left(x_{0}, t_{0}\right)-\phi\left(x_{0}, t_{0}\right)$. In addition, since $\underline{z}_{A}\left(x_{0}, t_{0}\right) \in(-A, 0), J_{A}^{\tau}\left(x_{\tau}, t_{\tau}\right) \in(-A, 0)$ for all $\tau$ sufficiently small.

Using the definition of $H^{\tau}$ and that $J^{\tau}$ satisfies $(1.9)$, when $\tau$ is sufficiently small, we obtain, at $\left(x_{\tau}, t_{\tau}\right)$,

$$
\frac{\tau}{1+\tau}-f_{S^{n-1}} \frac{d v}{\frac{1}{\tau}+\phi_{t}\left(x_{\tau}, t_{\tau}\right)+a_{n, \tau} v \cdot D \phi\left(x_{\tau}, t_{\tau}\right)} \geq 0 .
$$

We point out that we need only use this form of the Hamiltonian $H$ (3.4) since, for any fixed $p$, $\Phi(1)>\sqrt{n \tau}|p| /(1+\tau)$ when $\tau$ is small enough.

Dividing both sides by $\tau$ and using Taylor's theorem on the integrand, we see that, at $\left(x_{\tau}, t_{\tau}\right)$,

$$
0 \leq \frac{1}{1+\tau}-f\left(1-\tau \phi_{t}\left(x_{\tau}, t_{\tau}\right)-\sqrt{\tau n} v \cdot D \phi\left(x_{\tau}, t_{\tau}\right)+n \tau\left(v \cdot D \phi\left(x_{\tau}, t_{\tau}\right)\right)^{2}+\mathrm{o}(\tau)\right) .
$$


The third term under the integral vanishes after integrating over $S^{n-1}$ as it is odd in $v$, while, a calculation in the Appendix, yields that

$$
f_{S^{n-1}} n\left(v \cdot D \phi\left(x_{\tau}, t_{\tau}\right)\right)^{2} d v=\left|D \phi\left(x_{\tau}, t_{\tau}\right)\right|^{2} .
$$

Applying Taylor's theorem again, it is now immediate that, at $\left(x_{\tau}, t_{\tau}\right)$,

$$
0 \leq(1-\tau+\mathrm{o}(\tau))-1+\tau \phi_{t}\left(x_{\tau}, t_{\tau}\right)-\tau\left|D \phi\left(x_{\tau}, t_{\tau}\right)\right|^{2}+o(\tau),
$$

which, after dividing by $\tau$ and letting $\tau \rightarrow 0$, yields

$$
0 \leq \phi_{t}\left(x_{0}, t_{0}\right)-\left|D \phi\left(x_{0}, t_{0}\right)\right|^{2}-1 .
$$

The case when $t_{0}=0$ may be easily handled by a combination of the methods above and in Section 2 ,

\section{The failure of positivity}

\section{The reactive-telegraph equation: proof of Theorem 1.1 when $n=1$}

We prove that the reactive-telegraph equation preserves positivity when $n=1$. We note that the upper bound in the statement of Theorem 1.1 is clearly not sharp. Due to the lack of maximum principle and the structure of the equation (1.1), the upper and lower bounds must be obtained simultaneously. Since our main interest is in the lower bound, we do not optimize the proof of the upper bound and use 2 since it is sufficient to obtain the desired lower bound.

Proof of Theorem 1.1 when $n=1$. Before beginning we note that it is enough to prove the claim with nonlinearity $\rho(1-\rho)$, since $\rho(1-\rho) \leq \rho(1-\rho)_{+}$. The key tool is the equivalence between (1.1) and $(1.3)$ in one spatial dimension, which may also be written as 1.2 . That is, let $p^{ \pm}$solve (1.2) with $p^{+}(\cdot, 0)=p^{-}(\cdot, 0)=\rho_{0}$, then $\rho=\left(p^{+}+p^{-}\right) / 2$. Hence, if we show that $0 \leq p^{+}, p^{-} \leq 2$, the result follows for $\rho$.

Fix $\epsilon>0$. By approximation, we may assume that $p^{ \pm}$are smooth and uniformly equal to $2 \sqrt{\epsilon}$ outside a compact set and that the initial data satisfy $2 \sqrt{\epsilon} \leq \rho_{0} \leq 1$. We define

$$
p_{\epsilon}^{+}(x, t)=p^{+}(x, t)+\epsilon t \quad \text { and } \quad p_{\epsilon}^{-}(x, t)=p^{-}(x, t)+\epsilon t,
$$

and let

$$
T_{\epsilon}=\sup \left\{t \in(0,1 / \sqrt{\epsilon}): \sqrt{\epsilon}<p_{\epsilon}^{+}(x, s), p_{\epsilon}^{-}(x, s)<2-\sqrt{\epsilon} \text { for all } s \in(0, t)\right\} .
$$

Here, $T_{\epsilon}$ is the first time that either $p_{\epsilon}^{+}$or $p_{\epsilon}^{-}$"touches" $\sqrt{\epsilon}$ or $2-\sqrt{\epsilon}$. It is well-defined and positive due to the smoothness of $\rho_{0}$ that is inherited by $p_{\epsilon}^{+}$and $p_{\epsilon}^{-}$.

Our goal is to show that $T_{\epsilon}=1 / \sqrt{\epsilon}$ for every $\epsilon>0$. Once we have shown this, the bounds on $p^{+}$and $p^{-}$follow by taking $\epsilon \rightarrow 0$. To this end, we proceed by contradiction and assume that $T_{\epsilon} \in(0,1 / \sqrt{\epsilon})$.

Let $x_{\epsilon} \in \mathbb{R}$ be such that $p_{\epsilon}^{+}\left(x_{\epsilon}, T_{\epsilon}\right)$ is either $\sqrt{\epsilon}$ or $2-\sqrt{\epsilon}$. The argument follows similarly if $p_{\epsilon}^{-}\left(x_{\epsilon}, T_{\epsilon}\right)$ is either $\sqrt{\epsilon}$ or $2-\sqrt{\epsilon}$.

Before continuing with the proof, we briefly justify the existence of $x_{\epsilon}$. Let $R_{\epsilon}>0$ be sufficiently large so that $\rho_{0} \equiv 2 \sqrt{\epsilon}$ on $B_{R_{\epsilon}}^{c}$. Since 1.2 has no space or time dependence, the finite speed of 
propagation for kinetic equations implies that $p_{x}^{ \pm} \equiv 0$ on $B_{R_{\epsilon}+(\tau \epsilon)^{-1 / 2}}^{c} \times[0,1 / \sqrt{\epsilon}]$. As such, (1.2) reduces to an ordinary differential equation in $t$ at every point $x \in B_{R_{\epsilon}+(\tau \epsilon)^{-1 / 2}}^{c}$. The ODE is the same for both $p^{+}$and $p^{-}$, and, hence, the $p^{+}$and $p^{-}$are equal on $B_{R_{\epsilon}+(\tau \epsilon)^{-1 / 2}}^{c}$. This, in turn, implies that $p^{+}=p^{-}=\rho$ on $B_{R_{\epsilon}+(\tau \epsilon)^{-1 / 2}}^{c}$, which, from 1.2 , implies that

$$
\rho_{t}=\rho(1-\rho) \quad \text { with } \quad \rho_{0}(x)=2 \sqrt{\epsilon}
$$

on $B_{R_{\epsilon}+(\tau \epsilon)^{-1 / 2}}^{c} \times[0,1 / \sqrt{\epsilon}]$. At this point, it is clear that $p_{\epsilon}^{+}$and $p_{\epsilon}^{-}$can achieve neither the value $\sqrt{\epsilon}$ nor the value $2-\sqrt{\epsilon}$ on $B_{R_{\epsilon}+(\tau \epsilon)^{-1 / 2}}^{c} \times[0,1 / \sqrt{\epsilon}]$, so long as $\epsilon$ is sufficiently small. As such, if $T_{\epsilon}<1 / \sqrt{\epsilon}$, the smoothness of $p_{\epsilon}^{+}$and $p_{\epsilon}^{-}$guarantees the existence of $x_{\epsilon} \in B_{R_{\epsilon}+(\tau \epsilon)^{-1 / 2}}$.

First we consider the case that $p_{\epsilon}^{+}\left(x_{\epsilon}, T_{\epsilon}\right)=\sqrt{\epsilon}$. Using that $p_{\epsilon}^{+}$is smooth and $\left(x_{\epsilon}, T_{\epsilon}\right)$ is the location of a minimum of $p_{\epsilon}^{+}$on $\mathbb{R} \times\left(0, T_{\epsilon}\right]$, we see that, at $\left(x_{\epsilon}, T_{\epsilon}\right)$,

$$
\left(p_{\epsilon}^{+}\right)_{t}+\frac{1}{\sqrt{\tau}}\left(p_{\epsilon}^{+}\right)_{x} \leq 0 .
$$

From this, along with 1.2 and the definition of $p_{\epsilon}^{+}$in terms of $p^{+}$, we get that, at $\left(x_{\epsilon}, T_{\epsilon}\right)$,

$$
\epsilon+\frac{1}{\tau}\left(\rho-p^{+}\right)+\rho(1-\rho)=\left(p_{\epsilon}^{+}\right)_{t}+\frac{1}{\sqrt{\tau}}\left(p_{\epsilon}^{+}\right)_{x} \leq 0 .
$$

The definition of $T_{\epsilon}$ yields that $\sqrt{\epsilon} \leq p_{\epsilon}^{-}\left(x_{\epsilon}, T_{\epsilon}\right) \leq 2-\sqrt{\epsilon}$. Using these bounds for $p_{\epsilon}^{-}$, the facts that $p_{\epsilon}^{+}\left(x_{\epsilon}, T_{\epsilon}\right)=\sqrt{\epsilon}$ and $\epsilon T_{\epsilon}<\sqrt{\epsilon}$, and the relationship between $p_{\epsilon}^{ \pm}$and $p^{ \pm}$, we obtain

$$
0 \leq \rho\left(x_{\epsilon}, T_{\epsilon}\right) \leq 1
$$

Also, since $p_{\epsilon}^{+}\left(x_{\epsilon}, T_{\epsilon}\right) \leq p_{\epsilon}^{-}\left(x_{\epsilon}, T_{\epsilon}\right)$, it follows that $p^{+}\left(x_{\epsilon}, T_{\epsilon}\right) \leq \rho\left(x_{\epsilon}, T_{\epsilon}\right)$. Hence, at $\left(x_{\epsilon}, T_{\epsilon}\right)$,

$$
\frac{1}{\tau}\left(\rho-p^{+}\right)+\rho(1-\rho) \geq 0 .
$$

The combination of this inequality with the positivity of $\epsilon$ contradicts (6.1), finishing this case. Now we consider the case that $p_{\epsilon}^{+}\left(x_{\epsilon}, T_{\epsilon}\right)=2-\sqrt{\epsilon}$. Reasoning as above, we see that, at $\left(x_{\epsilon}, T_{\epsilon}\right)$,

$$
0 \leq\left(p_{\epsilon}^{+}\right)_{t}+\frac{1}{\sqrt{\tau}}\left(p_{\epsilon}^{+}\right)_{x}=\epsilon+\frac{1}{\tau}\left(\rho-p^{+}\right)+\rho(1-\rho) .
$$

Moving the $\epsilon$ and $p^{+}$terms from the right hand side to the left hand side and using that $p^{+}=p_{\epsilon}^{+}-\epsilon T_{\epsilon}$ and $T_{\epsilon}<1 / \sqrt{\epsilon}$, we obtain

$$
\frac{2}{\tau}-\frac{\sqrt{\epsilon}(2-\tau \sqrt{\epsilon})}{\tau}=\frac{1}{\tau}(2-2 \sqrt{\epsilon})-\epsilon \leq \frac{1}{\tau}\left(p_{\epsilon}^{+}-\epsilon T_{\epsilon}\right)-\epsilon \leq \frac{1}{\tau} \rho+\rho(1-\rho) .
$$

On the other hand the definition of $T_{\epsilon}$ implies that $\sqrt{\epsilon} \leq p_{\epsilon}^{-}\left(x_{\epsilon}, T_{\epsilon}\right) \leq 2-\sqrt{\epsilon}$. From this, we see that

$$
1-\sqrt{\epsilon} \leq \frac{\left(p_{\epsilon}^{+}\left(x_{\epsilon}, T_{\epsilon}\right)-\epsilon T_{\epsilon}\right)+\left(p_{\epsilon}^{-}\left(x_{\epsilon}, T_{\epsilon}\right)-T_{\epsilon}\right)}{2}=\rho\left(x_{\epsilon}, T_{\epsilon}\right)<\frac{p_{\epsilon}^{+}\left(x_{\epsilon}, T_{\epsilon}\right)+p_{\epsilon}^{-}\left(x_{\epsilon}, T_{\epsilon}\right)}{2} \leq 2-\sqrt{\epsilon},
$$

or, more succinctly,

$$
1-\sqrt{\epsilon} \leq \rho\left(x_{\epsilon}, T_{\epsilon}\right)<2-\sqrt{\epsilon} .
$$


When $\epsilon$ is sufficiently small, depending only on $\tau$, the two inequalities $(6.2)$ and $(6.3)$ are incompatible. Briefly, this may be seen by considering two cases. First, if $\rho \in[1-\epsilon, 3 / 2]$, we obtain

$$
\frac{2}{\tau}-\frac{\sqrt{\epsilon}(2-\tau \sqrt{\epsilon})}{\tau} \leq \frac{1}{\tau} \rho+\rho(1-\rho) \leq \frac{3}{2 \tau}+\frac{3}{2} \sqrt{\epsilon}
$$

from 6.2 . This is clearly a contradiction when $\epsilon$ is sufficiently small. Second, if $\rho \in[3 / 2,2-\sqrt{\epsilon}]$, we find from $(6.2)$

$$
\frac{2}{\tau}-\frac{\sqrt{\epsilon}(2-\tau \sqrt{\epsilon})}{\tau} \leq \frac{1}{\tau} \rho+\rho(1-\rho) \leq \frac{2-\sqrt{\epsilon}}{\tau}+\frac{3}{2}\left(1-\frac{3}{2}\right),
$$

which is also a contradiction for $\epsilon$ is sufficiently small.

This finishes the consideration of the second case and thus finishes the proof.

\section{The reactive-telegraph equation: proof of Theorem 1.1 when $n \geq 2$}

We need the following estimate:

Theorem 6.1 (Chapter 1, Theorem 4.1 [26]). Let $F_{\text {wave }}: \mathbb{R}^{2} \rightarrow \mathbb{R}$ be a smooth function such that $F_{\text {wave }}(0,0)=0$. Assume that $2 s \geq n+2, f \in H^{s+1}\left(\mathbb{R}^{n}\right)$, and $g \in H^{s}\left(\mathbb{R}^{n}\right)$. Then there exists $T>0$, depending on $F_{\text {wave }},\|f\|_{H^{s+1}}$, and $\|g\|_{H^{s}}$, such that the initial value problem

$$
\begin{cases}u_{t t}=\Delta u+F_{\text {wave }}\left(u, u_{t}\right) & \text { in } \mathbb{R}^{n} \times(0, T), \\ u=f, \quad u_{t}=g & \text { on } \mathbb{R}^{n} \times\{0\}\end{cases}
$$

has a unique solution u satisfying, for some universal constant $C$,

$$
\|u\|_{H^{s+1}\left(\mathbb{R}^{n} \times[0, T]\right)} \leq C\left(\|f\|_{H^{s+1}\left(\mathbb{R}^{n}\right)}+\|g\|_{H^{s}\left(\mathbb{R}^{n}\right)}\right) .
$$

With this in hand, we now show that the reactive-telegraph equation (1.1) does not preserve positivity.

Proof of Theorem 1.1 when $n \geq 2$. It is enough to work with $n=2$, since any example in this setting also works for $n>2$.

Fix $\rho_{0}(x)=\epsilon e^{-|x|^{2} / \delta}$ for $\epsilon, \delta \in(0,1)$ so that $\epsilon \leq \delta^{5 / 4}$ and let $\rho$ be the solution to

$$
\left\{\begin{array}{l}
\tau \rho_{t t}+(1-\tau+2 \tau \rho) \rho_{t}=\Delta \rho+\rho(1-\rho) \text { in } \mathbb{R}^{2} \times(0, T), \\
\rho(\cdot, 0)=\rho_{0} \text { and } \rho_{t}(\cdot, 0)=0 \text { on } \mathbb{R}^{2}
\end{array}\right.
$$

where $T>0$ gives the time interval of existence, which we obtain below.

Next we change variables to transform (6.4) to a wave equation with a right hand side which we can analyze using Theorem 6.1 .

It is immediate that $u(x, t)=e^{(1-\tau) t / 2 \tau} \rho(x, t)$ satisfies

$$
\tau u_{t t}-\Delta u=u\left(1-\frac{(1-\tau)^{2}}{4 \tau^{2}}+2 \tau e^{-(1-\tau) t / 2 \tau} u_{t}-e^{-(1-\tau) t / 2 \tau} u\right)
$$


Letting $v(x, t)=u(x, \sqrt{\tau} t)$, we find

$$
\left\{\begin{array}{l}
v_{t t}-\Delta v=v\left(1-\frac{(1-\tau)^{2}}{4 \tau^{2}}+2 \sqrt{\tau} e^{-(1-\tau) t / 2 \tau} v_{t}-e^{-(1-\tau) t / 2 \tau} v\right) \quad \text { on } \quad \mathbb{R}^{2} \times(0, T), \\
v(\cdot, 0)=\rho_{0} \quad \text { and } \quad v_{t}(\cdot, 0)=\frac{1-\tau}{2 \sqrt{\tau}} \rho_{0} \quad \text { on } \quad \mathbb{R}^{2}
\end{array}\right.
$$

Using Theorem 6.1 with $s=7 / 2$ and $s=5 / 2$ respectively, we find $T>0$ and $C_{\tau}$, which is independent of $T$ and will change from line to line below, such that

$$
\left\|v_{t}\right\|_{L^{\infty}\left((0, T) \times \mathbb{R}^{2}\right)} \leq C_{\mathrm{Sob}}\|v\|_{H^{7 / 2}\left((0, T) \times \mathbb{R}^{2}\right)} \leq C_{\tau}\left\|\rho_{0}\right\|_{H^{7 / 2}\left(\mathbb{R}^{2}\right)} \leq C_{\tau} \epsilon \delta^{-5 / 4}
$$

and

$$
\|v\|_{L^{\infty}\left((0, T) \times \mathbb{R}^{2}\right)} \leq C_{\mathrm{Sob}}\|v\|_{H^{5 / 2}\left((0, T) \times \mathbb{R}^{2}\right)} \leq C_{\tau}\left\|\rho_{0}\right\|_{\left.H^{5 / 2}\left(\mathbb{R}^{2}\right)\right)} \leq C_{\tau} \epsilon \delta^{-3 / 4} ;
$$

note that in the second inequality we absorbed $C_{\text {Sob }}$ into $C_{\tau}$ to simplify the notation.

Since $\epsilon \leq \delta^{5 / 4}$, it follows that, for some $C_{\tau}$ depending only on $\tau$,

$$
\left\|\rho_{0}\right\|_{H^{7 / 2}} \leq C_{\tau}
$$

In view of this and Theorem 6.1, it follows that $T$ does not depend on $\epsilon$ or $\delta$.

Duhamel's formula for the solution to the wave equation for $n=2$ gives

$$
\begin{aligned}
& v(x, t)=\frac{1}{2} f_{B_{t}(x)} \frac{1}{\sqrt{t^{2}-|x-y|^{2}}}\left[t^{2} \frac{1-\tau}{2 \sqrt{n \tau}} \rho_{0}(y)+t \rho_{0}(y)+t D \rho_{0}(y) \cdot(y-x)\right] d y \\
& \quad+\int_{0}^{t} s^{2} f_{B_{s}(x)} \frac{v}{\sqrt{s^{2}-|x-y|^{2}}}\left(1-\frac{(1-\tau)^{2}}{4 \tau^{2}}+2 \sqrt{\tau} e^{-(1-\tau) t / 2 \tau} v_{t}-e^{-(1-\tau) t / 2 \tau} v\right) d y d s .
\end{aligned}
$$

At this point we mention that this example does not work in one dimension because the general form of the solution is different in one dimension. In particular, the crucial term $D \rho_{0}$ does not appear.

Inserting the bounds (6.5) and 6.6 in (6.7), we find

$$
v(0, t) \leq \epsilon\left(e^{-t^{2} / \delta}\left[t \frac{|1-\tau|}{2 \sqrt{\tau}}+1-2 \frac{t^{2}}{\delta}\right]+C_{\tau} \delta^{-3 / 4} t^{2}\left[1+\epsilon \delta^{-5 / 4}\right]\right)
$$

which, for $\delta=t^{2}$, becomes

$$
v(0, t) \leq \epsilon\left(e^{-1} t \frac{|1-\tau|}{2 \sqrt{\tau}}-2 e^{-1}+C_{\tau} t^{1 / 2}+\epsilon C_{\tau} t^{-2}\right) .
$$

The claim follows by choosing first $t$ and then $\epsilon$ small to obtain $v(0, t)<0$, and, hence, that $\rho(0, t / \sqrt{\tau})<0$.

\section{A kinetic model that does not preserve positivity}

In the interest of simplicity, we study here a slightly different model than (1.3) and somewhat singular initial data. Generalizations to a larger class of equations and initial data are conceptually straightforward, though with significantly more involved computations. 
We consider solutions to a two-dimensional discrete version of 1.3 given by

$$
\left\{\begin{array}{l}
p_{t}+a_{2, \tau} v \cdot D p=\frac{1}{\tau}(\rho-p)+p(1-p) \quad \text { in } \quad \mathbb{R}^{2} \times S_{d}^{1} \times \mathbb{R}_{+}, \\
p(\cdot, 0)=p_{0} \quad \text { on } \quad \mathbb{R}^{2} \times S_{d}^{1}
\end{array}\right.
$$

where $S_{d}^{1}:=\left\{e_{1}, e_{2},-e_{1},-e_{2}\right\}$ with $e_{1}, e_{2}$ the standard basis vectors of $\mathbb{R}^{2}$ and

$$
\rho(x, t):=\frac{1}{4}\left(\sum_{v \in S_{d}^{1}} p(x, v, t)\right)
$$

We have:

Proposition 6.2. Fix $\tau>4$. There exist a bounded $p_{0}$ with $\left(\sum_{v \in S_{d}^{1}} p_{0}\right) / 4 \leq 1$ and $\left(x_{0}, v_{0}, t_{0}\right) \in$ $\mathbb{R}^{2} \times S_{d}^{1} \times \mathbb{R}_{+}$such that, if $p$ solves (6.8) with initial datum $p_{0}$, then $p\left(x_{0}, v_{0}, t_{0}\right)<0$.

The idea is to choose $p_{0}$ having three patches, one moving in the $-e_{1}$ direction, one moving in the $e_{2}$ direction, and another moving in the $e_{1}$ direction, which will eventually collide. Their sum will then be large enough to force the reaction term to be negative, which will cause the population density of the species moving in the $e_{2}$ direction to become negative.

Proof of Proposition 6.2. To choose $p_{0}$, we introduce the (moving) sets

$$
\left\{\begin{array}{l}
X_{1, t}=\left\{\left(x_{1}, x_{2}\right) \in \mathbb{R}^{2}:\left|x_{2}\right|<-x_{1}+a_{2, \tau} t\right\}, \quad X_{2, t}=\left\{\left(x_{1}, x_{2}\right) \in \mathbb{R}^{2}:\left|x_{1}\right|<x_{2}+a_{2, \tau} t\right\}, \quad \text { and } \\
X_{3, t}=\left\{\left(x_{1}, x_{2}\right) \in \mathbb{R}^{2}:\left|x_{2}\right|<x_{1}+a_{2, \tau} t\right\}
\end{array}\right.
$$

which, when $t=0$, are disjoint cones with a vertex at the origin in the directions $-e_{1}, e_{2}$, and $e_{1}$, respectively, while, for $t>0$, they move in time in the directions $e_{1},-e_{2}$, and $-e_{1}$, respectively, with speed $a_{2, \tau}$.

Let $p_{0}$ be so that

$$
\left\{\begin{array}{l}
p_{0}\left(x, e_{2}\right) \equiv 0, \quad p_{0}\left(x, e_{1}\right)=4\left(1-\frac{3}{\tau}\right) \mathbb{1}_{X_{1,0}}(x), \\
p_{0}\left(x,-e_{2}\right)=4\left(1-\frac{3}{\tau}\right) \mathbb{1}_{X_{2,0}}(x), \quad \text { and } \quad p_{0}\left(x,-e_{1}\right)=4\left(1-\frac{3}{\tau}\right) \mathbb{1}_{X_{3,0}}(x) .
\end{array}\right.
$$

The constant $4(1-3 / \tau)$ is chosen so that $p_{0}$ is in a quasi-equilibrium, that is, for any fixed $e \in S_{d}^{1}$, the right hand side of 6.8 is zero at $t=0$.

A simple computation shows that, in view of the choice of the sets $X_{i, 0}$,

$$
0 \leq \rho(\cdot, 0) \leq 1 \text { in } \mathbb{R}^{2}
$$

To simplify the notation, we introduce

$$
\bar{p}(x, t):=e^{-t / \tau} p\left(x+e_{2} a_{2, \tau} t, e_{2}, t\right),
$$

which satisfies

$$
\bar{p}_{t}=\frac{1}{\tau} \bar{\rho}+\bar{\rho}(1-\bar{\rho}) \quad \text { in } \quad \mathbb{R}^{2} \times S_{d}^{1} \times \mathbb{R}_{+},
$$

with $\bar{\rho}(x, t)=\rho\left(x+e_{2} a_{2, \tau} t, t\right)$. For future reference note that the right hand side of 6.9$)$ is decreasing in $\bar{\rho}$ when $\tau>1$ and $\bar{\rho} \geq(\tau+1) / 2 \tau$. 
Fix $\epsilon>0$. It is easy to see, from equation (6.8), that there exists $T_{\epsilon}$, depending only on $\epsilon$, such that, for $t \in\left(0, T_{\epsilon}\right)$,

$$
\begin{aligned}
& 4\left(1-\frac{3}{\tau}\right) \mathbb{1}_{X_{1, t}}(x) \geq p\left(x, e_{1}, t\right) \geq 4(1-\epsilon)\left(1-\frac{3}{\tau}\right) \mathbb{1}_{X_{1, t}}(x), \\
& 4\left(1-\frac{3}{\tau}\right) \mathbb{1}_{X_{2, t}}(x) \geq p_{0}\left(x,-e_{2}\right) \geq 4(1-\epsilon)\left(1-\frac{3}{\tau}\right) \mathbb{1}_{X_{2, t}}(x), \quad \text { and } \\
& 4\left(1-\frac{3}{\tau}\right) \mathbb{1}_{X_{3, t}}(x) \geq p\left(x,-e_{1}, t\right) \geq 4(1-\epsilon)\left(1-\frac{3}{\tau}\right) \mathbb{1}_{X_{3, t}}(x) .
\end{aligned}
$$

Fix $\delta \in\left(0,2 T_{\epsilon} a_{2, \tau}\right)$. We point out that the domains of the indicator functions in 6.10$)$ are initially disjoint but contain $(0,-\delta)$ when $t>\delta / 2 a_{2, \tau}$. Then 6.10 yields, for all $t \in\left(\delta / 2 a_{2, \tau}, T_{\epsilon}\right)$,

$$
\bar{\rho}((0,-\delta), t) \geq 3(1-\epsilon)\left(1-\frac{3}{\tau}\right)
$$

and, hence, for $\epsilon$ small enough and $t>\delta / 2 a_{2, \tau}$ and since $\tau>4$,

$$
\bar{\rho}((0,-\delta), t) \geq(\tau+1) / 2 \tau .
$$

Using 6.12, the lower bound in 6.11), and the fact that $\tau>4$ and choosing an even smaller $\epsilon$ in (6.9), we obtain, for all $t \in\left(\delta / 2 a_{2, \tau}, T_{\epsilon}\right)$,

$$
\begin{aligned}
\bar{p}_{t}((0,-\delta), t) & =\frac{1}{\tau} \bar{\rho}((0,-\delta), t)+\bar{\rho}((0,-\delta), t)(1-\bar{\rho}((0,-\delta), t)) \\
& \leq \frac{3(1-\epsilon)}{\tau}\left(1-\frac{3}{\tau}\right)+3(1-\epsilon)\left(1-\frac{3}{\tau}\right)\left(1-3(1-\epsilon)\left(1-\frac{3}{\tau}\right)\right) \\
& =3(1-\epsilon)\left(1-\frac{3}{\tau}\right)\left(1+\frac{1}{\tau}-3(1-\epsilon)\left(1-\frac{3}{\tau}\right)\right)<0
\end{aligned}
$$

By a similar, though simpler, computation, $\bar{p}_{t}((0,-\delta), t)=0$ when $t \in\left[0, \delta / 2 a_{2, \tau}\right]$. Using this, 6.13), and that $\bar{p}((0,-\delta), 0)=0$, we see that, for all $t \in\left(\delta / 2 a_{2, \tau}, T_{\epsilon}\right)$,

$$
p\left(\left(a_{2, \tau} t-\delta\right) e_{2}, e_{2}, t\right)=\bar{p}((0,-\delta), t)<0
$$

and the proof is now complete.

\section{A Appendix: The integrals (3.3) and (5.5)}

We compute the three simple integrals used above.

The integral 3.3

When $n=1$, this computation is simple, since it reduces to a sum. On the other hand, when $n>3$, the expression becomes more complicated. As such, we omit these cases. Here, for $s>1$, $w \in S^{n-1}$, and $n=2,3$, we compute the integral

$$
\Phi(s)=f_{S^{n-1}} \frac{d v}{s+v \cdot w} .
$$


When $n=2$, changing variables we may assume that $w=(|w|, 0)$. This, along with using spherical coordinates, yields the integral

$$
\Phi(s)=f_{S^{1}} \frac{d v}{s+v \cdot w}=\frac{1}{\pi} \int_{0}^{\pi} \frac{d \theta}{s+\cos (\theta)} .
$$

The substitution $r=\tan (\theta / 2)$ then implies

$$
\Phi(s)=\frac{2}{\pi} \int_{0}^{\infty} \frac{d r}{2\left(1+r^{2}\right)+\left(1-r^{2}\right)}=\frac{2}{\pi} \int_{0}^{\infty} \frac{d r}{s+1+(s-1) r^{2}}=\frac{1}{\sqrt{s^{2}-1}}
$$

When $n=3$, again we change variables so that $v \cdot w=v_{1}|w|$. Then, using spherical coordinates, A.1 becomes

$$
\Phi(s)=f_{S^{2}} \frac{d v}{a+v \cdot w}=\frac{1}{2} \int_{0}^{\pi} \frac{\sin (\theta) d \theta}{a+|w| \cos (\theta)} .
$$

The substitution $r=\tan (\theta / 2)$ yields

$$
\begin{aligned}
\Phi(s) & =2 \int_{0}^{\infty} \frac{r}{(2+1)+2 s r^{2}+(s-1) r^{4}} d r=\frac{2}{s-1} \int_{0}^{\infty} \frac{r}{\left(r^{2}+1\right)\left(r^{2}+\frac{s+1}{s-1}\right)} d r \\
& =\int_{0}^{\infty}\left[\frac{r}{r^{2}+1}-\frac{r}{r^{2}+\frac{s+1}{s-1}}\right] d r=\frac{1}{2} \log \left(\frac{s+1}{s-1}\right) .
\end{aligned}
$$

\section{The convergence of the integral in 3.30 to infinity}

We prove that the integral term in 3.30 tends to infinity as $\mu \rightarrow \mu_{c}^{-}$where $\mu_{c}:=(n-1) / 2$.

Following the work above, we have, for some normalizing factor $\omega_{n}$ depending only on $n$,

$$
f_{S^{n-1}} \frac{d v}{\left(1+v_{1}\right)^{\mu}}=\frac{1}{\omega_{n}} \int_{S^{n-1}} \frac{\sin (\theta)^{n-2}}{(1+\cos (\theta))^{\mu}} d \theta
$$

and the change of variables $r=\tan (\theta / 2)$ yields

$$
f_{S^{n-1}} \frac{d v}{\left(1+v_{1}\right)^{\mu}}=\frac{1}{\omega_{n}} \int_{0}^{\infty} \frac{\frac{(2 r)^{n-2}}{\left(1+r^{2}\right)^{n-2}}}{\left(1+\frac{1-r^{2}}{1+r^{2}}\right)^{\mu}} \frac{2 d r}{1+r^{2}}=\frac{2^{n-1-\mu}}{\omega_{n}} \int_{0}^{\infty} \frac{r^{n-2}}{\left(1+r^{2}\right)^{n-1-\mu}} d r .
$$

This integral is finite only when $\mu<(n-1) / 2$. When $\mu=1$, the integral is finite if and only if $n>3$. Hence, $\Phi(1)<\infty$ if and only if $n>3$.

The integral (5.5)

We show here that, for any vector $w \in S^{n-1}$,

$$
f_{S^{n-1}} n(v \cdot w)^{2} d v=1
$$

The general result follows by scaling. Moreover, we consider only the case $n>2$, since, for $n=1,2$ the formula is almost immediate. 
Changing variables so that the term $v \cdot w=v_{1}$ gives,

$$
f_{S^{n-1}}(v \cdot w)^{2} d v=f_{S^{n-1}} v_{1}^{2} d v_{1}
$$

Using polar coordinates, we obtain

$$
\begin{aligned}
f_{S^{n-1}}(v \cdot w)^{2} d v & =\frac{1}{\int_{0}^{\pi} \sin (\theta)^{n-2} d \theta} \int_{0}^{\pi} \sin (\theta)^{n-2} \cos (\theta)^{2} d \theta \\
& =\frac{1}{\int_{0}^{\pi} \sin (\theta)^{n-2} d \theta} \int_{0}^{\pi}\left(\sin (\theta)^{n-2}-\sin (\theta)^{n}\right) d \theta=1-\frac{\int_{0}^{\pi} \sin (\theta)^{n} d \theta}{\int_{0}^{\pi} \sin (\theta)^{n-2} d \theta} .
\end{aligned}
$$

Denoting $I_{n}=\int_{0}^{\pi} \sin (\theta)^{n} d \theta$, which is sometimes referred to as the Wallis integral, we integrate by parts to obtain the recurrence relation

$$
I_{n}=I_{n-2}-\int_{0}^{\pi} \sin (\theta)^{n-2} \cos (\theta)^{2} d \theta=I_{n-2}-\frac{1}{n-1} I_{n}
$$

which implies that $I_{n} / I_{n-2}=\frac{n-1}{n}$, and, hence,

$$
f_{S^{n-1}}(v \cdot w)^{2} d v=1-\frac{I_{n}}{I_{n-2}}=\frac{1}{n}
$$

\section{References}

[1] G. Barles. Nonlinear Neumann boundary conditions for quasilinear degenerate elliptic equations and applications. J. Differential Equations, 154(1):191-224, 1999.

[2] G. Barles, L. C. Evans, and P. E. Souganidis. Wavefront propagation for reaction-diffusion systems of PDE. Duke Math. J., 61(3):835-858, 1990.

[3] G. Barles and B. Perthame. Exit time problems in optimal control and vanishing viscosity method. SIAM J. Control Optim., 26(5):1133-1148, 1988.

[4] G. Barles and P. E. Souganidis. A remark on the asymptotic behavior of the solution of the KPP equation. C. R. Acad. Sci. Paris Sér. I Math., 319(7):679-684, 1994.

[5] E. Bouin and N. Caillerie. Spreading in kinetic reaction-transport equations in higher velocity dimensions. Preprint, 2017. https://arxiv.org/abs/1705.02191.

[6] E. Bouin and V. Calvez. A kinetic eikonal equation. C. R. Math. Acad. Sci. Paris, 350(56):243-248, 2012.

[7] E. Bouin, V. Calvez, E. Grenier, and G. Nadin. Large deviations for velocity-jump processes and non-local Hamilton-Jacobi equations. Preprint, 2017. https://arxiv.org/abs/1607.03676.

[8] E. Bouin, V. Calvez, and G. Nadin. Hyperbolic traveling waves driven by growth. Math. Models Methods Appl. Sci., 24(6):1165-1195, 2014.

[9] E. Bouin, V. Calvez, and G. Nadin. Propagation in a kinetic reaction-transport equation: travelling waves and accelerating fronts. Arch. Ration. Mech. Anal., 217(2):571-617, 2015. 
[10] M. Bramson. Convergence of solutions of the Kolmogorov equation to travelling waves. Mem. Amer. Math. Soc., 44(285):iv+190, 1983.

[11] X. Cabré and J.-M. Roquejoffre. The influence of fractional diffusion in Fisher-KPP equations. Comm. Math. Phys., 320(3):679-722, 2013.

[12] N. Caillerie. Large deviations of a velocity jump process with a Hamilton-Jacobi approach. $C$. R. Math. Acad. Sci. Paris, 355(2):170-175, 2017.

[13] M. G. Crandall, P.-L. Lions, and P. E. Souganidis. Maximal solutions and universal bounds for some partial differential equations of evolution. Arch. Rational Mech. Anal., 105(2):163-190, 1989.

[14] C. M. Cuesta, S. Hittmeir, and C. Schmeiser. Traveling waves of a kinetic transport model for the KPP-Fisher equation. SIAM J. Math. Anal., 44(6):4128-4146, 2012.

[15] L. C. Evans. The perturbed test function method for viscosity solutions of nonlinear PDE. Proc. Roy. Soc. Edinburgh Sect. A, 111(3-4):359-375, 1989.

[16] L. C. Evans and P. E. Souganidis. A PDE approach to geometric optics for certain semilinear parabolic equations. Indiana Univ. Math. J., 38(1):141-172, 1989.

[17] S. Fedotov. Wave front for a reaction-diffusion system and relativistic Hamilton-Jacobi dynamics. Phys. Rev. E (3), 59(5, part A):5040-5044, 1999.

[18] T. Hillen and H. G. Othmer. The diffusion limit of transport equations derived from velocityjump processes. SIAM J. Appl. Math., 61(3):751-775, 2000.

[19] E. E. Holmes. Are diffusion models too simple? a comparison with telegraph models of invasion. The American Naturalist, 142(5):779-795, 1993.

[20] W. Horsthemke. Spatial instabilities in reaction random walks with direction-independent kinetics. Physical Review E, 60(3):2651, 1999.

[21] M. Kac. A stochastic model related to the telegrapher's equation. Rocky Mountain J. Math., 4:497-509, 1974. Reprinting of an article published in 1956, Papers arising from a Conference on Stochastic Differential Equations (Univ. Alberta, Edmonton, Alta., 1972).

[22] P.-L. Lions. Generalized solutions of Hamilton-Jacobi equations, volume 69 of Research Notes in Mathematics. Pitman (Advanced Publishing Program), Boston, Mass.-London, 1982.

[23] A. J. Majda and P. E. Souganidis. Large-scale front dynamics for turbulent reaction-diffusion equations with separated velocity scales. Nonlinearity, 7(1):1-30, 1994.

[24] V. Méndez, J. Fort, and J. Farjas. Speed of wave-front solutions to hyperbolic reaction-diffusion equations. Physical Review E, 60(5):5231, 1999.

[25] V. Ortega-Cejas, J. Fort, and V. Méndez. The role of the delay time in the modeling of biological range expansions. Ecology, 85(1):258-264, 2004.

[26] C. D. Sogge. Lectures on nonlinear wave equations. Monographs in Analysis, II. International Press, Boston, MA, 1995. 
[27] A. Zlatoš. Sharp transition between extinction and propagation of reaction. J. Amer. Math. Soc., 19(1):251-263, 2006. 